\title{
1 miniTurbo-based interactomics of two plasma 2 membrane-localized SNARE proteins in 3 Marchantia polymorpha
}

4 Katharina Melkonian ${ }^{1}$, Sara Christina Stolze ${ }^{2}$, Anne Harzen ${ }^{1,2}$

5 and Hirofumi Nakagami ${ }^{1,2^{*}}$

6

$7 \quad{ }^{1}$ Basic Immune System of Plants, Max-Planck Institute for

8 Plant Breeding Research, Carl-von-Linné-Weg 10, 50829

9 Cologne, Germany

$10{ }^{2}$ Protein Mass Spectrometry, Max-Planck Institute for Plant

11 Breeding Research, Carl-von-Linné-Weg 10, 50829 Cologne,

12 Germany

\section{*Corresponding author:}

16 e-mail: nakagami@mpipz.mpg.de

17 Telephone: +49-221-5062-224

18 Fax: +49-221-5062-353

21 Total word count: 6769

22 Introduction: 1195

23 Materials and Methods: 2995

24 Results: 1451

25 Discussion: 1128

26 Acknowledgements: 42

27 Number of figures: 4

28 Coloured figures: 4

29 Tables: 1

30 Supporting information: 6 figures, 4 data tables 
34

35

57

58

59

60

61

62

\section{Summary}

- Marchantia polymorpha is a model liverwort and its overall low genetic redundancy is advantageous for dissecting complex pathways. Proximity-dependent in vivo biotin-labelling methods have emerged as powerful interactomics tools in recent years. However, interactomics studies applying proximity labelling are currently limited to angiosperm species in plants.

- Here, we established and evaluated a miniTurbo-based interactomics method in $M$. polymorpha using MpSYP12A and MpSYP13B, two plasma membranelocalized SNARE proteins, as baits.

- We show that our method yields a manifold of potential interactors of MpSYP12A and MpSYP13B compared to a co-immunoprecipitation approach. Our method could capture specific candidates for each SNARE.

- We conclude that a miniTurbo-based method is a feasible tool for interactomics in $M$. polymorpha and potentially applicable to other model bryophytes. Our interactome dataset on MpSYP12A and MpSYP13B will be a useful resource to elucidate the evolution of SNARE functions.

Keywords: interactomics, Marchantia polymorpha, 59 membrane-trafficking, miniTurbo, proximity labelling, SNARE 60 protein 


\section{Introduction}

The liverwort Marchantia polymorpha is a wellestablished model plant. The M. polymorpha genome has been sequenced (Bowman et al., 2017); (Montgomery et al., 2020) and genetic tools have been developed (Ishizaki et al., 2008; Ishizaki et al., 2013; Kubota et al., 2013; Ishizaki et al., 2015). In $M$. polymorpha, there is no evidence for whole genome duplication during evolution and the number of paralogs for many regulatory genes is rather low in comparison to other model plants (Bowman et al., 2017). Accordingly, low genetic redundancy is a useful feature of $M$. polymorpha in dissecting basic mechanisms and gene functions underlying complex pathways.

Elucidating complex pathways and protein-interaction networks remain major challenges in plant research. Conventional approaches to study protein-protein interactions, like co-immunoprecipitation (Co-IP) followed by mass spectrometry (MS) have limitations. Successful enrichment and purification under non-physiological conditions require a certain binding affinity between interactors. Therefore, Co-IP is often effective to capture stable complexes, while weak and transient associations can easily be lost. The analysis of interactions between members of subcellular proteomes may require an enrichment of cellular compartments before Co-IP to avoid artificial interactions upon cell lysis. In this context, proximity-dependent in vivo labelling (PL) approaches are gaining an increasing importance as alternative interactomics approaches.

The miniTurbo-based PL method was initially established in bacterial and mammalian cells, and is based on biotin ligase-mediated labelling of interaction partners with exogenously applied biotin (Branon et al., 2018). TurboID is a promiscuous biotin ligase that has been engineered from $E$. coli 
96 BirA with 15 mutations and has a higher ligase activity at a

97 wide range of temperatures compared to BirA. The miniTurbo is a smaller version of TurboID with a deleted N-terminal domain and has 13 mutations compared to BirA. TurboID and miniTurbo were reported to have higher activities than other biotin-ligases, namely BioID, BioID2, and BASU, in HEK 293T cells. Compared to TurboID, miniTurbo was found to be overall 1.5 times less active and showed a lower background labelling activity without exogenous application of biotin in HEK 293T cells (Branon et al., 2018). For PL of interaction partners, the biotin ligase is genetically fused to a bait. Molecules in proximity of the bait are biotinylated by the ligase in the presence of biotin. After labelling, biotinylated proteins can be extracted and enriched by streptavidin-pulldown before an identification by MS.

For pulldown of biotinylated proteins, it is not required 112 that proteins and complexes remain in their native state. 113 Because enrichment of biotinylated proteins does not rely on affinity to the bait proteins. PL approaches can thus also capture weak or transient interactions. The binding affinity between biotin and streptavidin is high. Therefore, protein extraction, binding, and washing steps can be conducted in the presence of high concentrations of detergents. The ligase activity can be inactivated during protein extraction, and thus artificial labelling upon cell lysis will not occur. This is advantageous for investigating interactions of proteins in subcellular compartments, including plasma membranelocalized proteins.

Since biotin ligases do not distinguish between real interaction partners and other molecules residing in proximity of the bait by chance, a certain level of unspecific labelling is expected. Unspecific labelling may occur according to an expression of the bait in a specific cellular compartment, cell-

129 type, tissue, organ, at a specific developmental stage, or 
130 physiological status and will be enhanced under saturating

131 labelling conditions. Therefore, non-saturating labelling

132 conditions are desirable and appropriate controls should be

133 designed to narrow down candidates of high confidence by

134 minimizing false positive identifications (Mair et al., 2019).

135 Plants, unlike animals, can synthesise biotin de novo

136 (Baldet et al., 1993; Baldet et al., 1997). High levels of

137 endogenous biotin may lead to background labelling,

138 potentially interfering with PL approaches. The endogenous

139 biotin level in M. polymorpha has not been investigated. In $A$.

140 thaliana, the sucrose- $\mathrm{H}^{+}$symporter AtSUC5 was identified to

141 mediate uptake of exogeneous biotin (Ludwig et al., 2000;

142 Pommerrenig et al., 2013). Until to date, AtSUC5 remains the

143 only sucrose transporter that was shown to function in biotin

144 uptake in planta. It is not yet known whether comparable

145 mechanisms for biotin uptake exist in M. polymorpha.

146 The TurboID or miniTurbo method has been

147 successfully applied in A. thaliana, Nicotiana benthamiana,

148 and Solanum lycopersicum using stable transgenic lines or

149 transient expression systems, to efficiently label and identify

150 subcellular proteomes in specific cell types (Mair et al., 2019)

151 as well as interacting partners of cytosolic (Zhang et al., 2019;

152 Arora et al., 2020) and nuclear (Mair et al., 2019) bait proteins

153 by MS. Arora et al. (2020) demonstrated that TurboID can be

154 applied to detect known interactions of plasma membrane-

155 localized proteins with a targeted approach using

156 immunoblotting. A direct comparison of interactomics applying

157 Co-IP and PL using the same plant materials is still missing. It

158 remains unclear whether biotin ligase-mediated PL approaches

159 can be sensitive and specific to reveal differences in

160 interactomes of very similar proteins, like closely related

161 homologs. Lastly, in bryophyte species, an interactome study

162 utilizing PL approaches has not yet been reported. 

ethylmaleimide-sensitive factor attachment protein receptor) proteins, MpSYP12A and MpSYP13B, are plasma membranelocalized and ubiquitously expressed throughout the thallus (Kanazawa et al., 2016; Kanazawa et al., 2020). Plant SNAREs modulate membrane-trafficking, intra- and intercellular signalling, and transport. In A. thaliana, 65 SNARE proteins have been identified, 9 of which are SYP1 family proteins that are plasma membrane-localized (Uemura et al., 2004). During land plant evolution, the expansion of SNARE proteins and their functional diversification was hypothesized to be linked to multicellularity and likely facilitated the adaptation to a terrestrial lifestyle (Sanderfoot, 2007). Thus, land plant secretory pathways are highly sophisticated, dynamic, and diversely regulated, being involved in a manifold of cellular processes ranging from polarized growth to defence responses (Batoko \& Moore, 2001; Collins et al., 2003; Catalano et al., 2007; Enami et al., 2009; Silva et al., 2010; Reichardt et al., 2011; Uemura et al., 2012; Ichikawa et al., 2014; Johansson et al., 2014; Yun et al., 2016; Xia et al., 2019; Hirano et al., 2020; Rubiato et al., 2021). In M. polymorpha, the SYP1 protein family is comprised of 4 members: SYP12A, SYP12B, SYP13A, and SYP13B. MpSYP13A and MpSYP13B belong to the SYP13 group and are closely related to AtSYP131 and AtSYP132. MpSYP12A and MpSYP12B belong to the SYP11/12 group, which is phylogenetically separated from the SYP11 or SYP12 group proteins in angiosperms (Kanazawa et al., 2016; Bowman et al., 2017). Carella et al. (2018) reported that MpSYP13B accumulated in haustoria-like structures upon infection of $M$. polymorpha with the oomycete pathogen Phytophthora palmivora. Recently, MpSYP12A was shown to localize to the phragmoplast during cell plate formation (Kanazawa et al., 2020). Interaction partners of SYP1 family proteins in $M$. polymorpha have not yet been identified. 
197 In this study, we established a miniTurbo-based PL

198 method for interactome profiling in M. polymorpha. Using

199 MpSYP12A and MpSYP13B as baits, we evaluated biotin-

200 labelling conditions and a procedure to enrich biotinylated

201 proteins, and then potential interactors were identified by MS.

202 We directly compared the performances of Co-IP and PL

203 approaches using the same plant materials. Lastly, by

204 comparing the identified interactomes of MpSYP12A and

$205 \mathrm{MpSYP13B}$, we found potential interactors that are specific to

206 each SNARE.

207

208 


\section{Materials and Methods}

210 Construction and cloning. Gateway entry vectors containing

211 genomic sequences for an expression of MpSYP12A and

212 MpSYP13B under their own regulatory elements (5'- and 3'-

213 flanking sequences and introns) in $M$. polymorpha were

214 provided by Takashi Ueda (Kanazawa et al., 2016). For N-

215 terminal tagging of MpSYP12A and MpSYP13B with

216 miniTurbo and Myc-tag, entry vector backbones were

217 linearized by restriction with SmaI or BamHI enzymes,

218 respectively. Codon-optimized miniTurbo (Fig. S1) was

219 synthesized by Thermo GeneArt and PCR-amplified from a

220 donor plasmid. Gateway entry vectors pMKMM20 (3.5 kb

221 upstream SYP13B:5'UTR:miniTurbo-Myc-SYP13B:3'UTR)

222 and pMKMM21 (3.5 kb upstream SYP12A:5'UTR:miniTurbo-

223 Myc-SYP12A:3'UTR) were generated by in-fusion cloning (HD

224 enzyme mix; Takara Bio). Gateway binary vectors pMKMM22

225 and pMKMM23 for the expression of miniTurbo-Myc-

226 MpSYP13B and miniTurbo-Myc-MpSYP12A were generated

227 by LR-recombination (LR clonase; Invitrogen) of pMKMM20

228 or pMKMM21 with pMpGWB301 (Fig. S2).

229

230

231

232

233

234

235

236

237

238

239

240

M. polymorpha lines used in this study. The male $M$. polymorpha Tak-1 ecotype was used as a wildtype. Transgenic lines in Tak-1 background were generated using the cut thallus method (Kubota et al., 2013) and Agrobacterium strain GV3101 carrying pMKMM22 or pMKMM23. Transformants were selected using Chlorsulfuron and Cefotaxime antibiotics for two generations. Selected transformants were screened for an expression of miniTurbo-Myc-MpSYP12A and miniTurboMyc-MpSYP13B fusion-proteins by immunoblotting. Transgenic lines were chosen that displayed similar expression levels of miniTurbo-Myc-MpSYP12A and miniTurbo-MycMpSYP13B for further analyses (Fig. S3a). M. polymorpha 
241 plants were grown and maintained on Gamborg's B5 (Duchefa;

242 G0209) half strength solid medium containing $1 \%$ plant agar in

243 a walk-in growth chamber under constant white light (50-60

$244 \mu$ mol photons LED $\mathrm{m}^{-2} \mathrm{~s}^{-1}$ ) at $22-24{ }^{\circ} \mathrm{C}$.

245 Sample preparation for PL and Co-IP experiments. $M$.

246 polymorpha Tak-1 and transgenic lines were grown from single

247 gemmae on Gamborg's B5 half strength solid medium

248 containing $0.8 \%$ plant agar for 10 days under constant white

249 light (50-60 $\mu \mathrm{mol}$ photons LED m $\left.\mathrm{s}^{-1}\right)$ at $22-24{ }^{\circ} \mathrm{C}$. Ten

250 individual 10-day old thalli were pooled per sample. For Co-IP,

251 untreated thalli were sampled immediately and frozen in liquid

252 nitrogen until further processing. For biotin treatment, thalli

253 were transferred into transparent 6- or 12-well plates (Greiner

254 Bio-One; 657160) and submerged in 0-700 $\mu \mathrm{M}$ biotin (Sigma;

255 B4501) solution in water. The thalli were vacuum-infiltrated

256 with biotin solutions for 5 minutes using a desiccator and the

257 samples were incubated for 0-24 hours at room temperature

$258\left(\mathrm{RT} ; 22-25^{\circ} \mathrm{C}\right)$ while shaking. After incubation, the thalli were

259 washed once with ice-cold ultrapure water for 2 minutes to

260 remove excess biotin. For sampling, thalli were transferred onto

261 filter paper (Whatman; 1001-085) and left for 10 seconds to

262 drain off excess liquid. Next, the thalli were pressed onto the

263 filter paper for 5 seconds, immediately transferred to fresh

264 tubes containing 2 stainless-steel beads and snap-frozen in

265 liquid nitrogen. The plant material was ground in liquid

266 nitrogen using a mixing mill (MM400, Retsch) for 5 minutes at

$26730 \mathrm{~Hz}$. For Co-IP, all following steps were conducted at $4{ }^{\circ} \mathrm{C}$.

268 The finely ground powder was mixed with extraction buffer (50

$269 \mathrm{mM}$ Tris pH 7.5, $150 \mathrm{mM} \mathrm{NaCl}, 10 \%$ glycerol, $2 \mathrm{mM}$

270 ethylenediaminetetraacetic acid (EDTA), $5 \mathrm{mM}$ dithiothreitol

271 (DTT), 1 \% Triton X-100, 1 \% Plant Protease Inhibitor; Sigma

272 P9599) and incubated for 30 minutes for protein extraction. For

273 PL samples, the powder was mixed with $500 \mu \mathrm{l}$ pre-heated 
274 SDT buffer (100 mM Tris $\mathrm{pH}$ 7.5, $4 \%$ sodium dodecyl sulfate

275 (SDS), $0.1 \mathrm{M}$ DTT) at $95{ }^{\circ} \mathrm{C}$ for 5 minutes and then sonicated

276 for 10 minutes. Cell debris was removed from the extracts by

277 two consecutive centrifugation steps (10,000 g, 10 minutes).

278 The protein concentration in cell extracts for Co-IP and PL was

279 determined using the Pierce $660 \mathrm{~nm}$ Assay (Thermo Fisher;

280 22660). Cell extracts with $500 \mu \mathrm{g}$ of total protein were used as

281 input for Myc-Trap Co-IP and biotin depletion before affinity-

282 pulldown of biotinylated proteins. For biotin-treated thalli, an

283 aliquot of each sample was taken for immunoblotting and

284 whole proteome analysis. Remaining samples were subjected to

285 biotin depletion before pulldown using streptavidin. Biotin

286 dilution series and time course experiments were each done in

287 two independent experiments (Fig. 1c, d; Fig. S4a, b).

288

289 Co-IP. Myc-Trap beads (Chromotek; ytma-20) were

290 equilibrated in ice-cold wash buffer (50 mM Tris $\mathrm{pH} 7.5,150$

$291 \mathrm{mM} \mathrm{NaCl}, 10 \%$ glycerol, $2 \mathrm{mM}$ EDTA) according to the

292 manufacturer's instructions. Cell extracts with $500 \mu \mathrm{g}$ of total

293 protein in $1 \mathrm{ml}$ volume were mixed with $25 \mu 1$ of equilibrated

294 Myc-Trap beads and pulldown was performed for 2 hours at

$2954{ }^{\circ} \mathrm{C}$ on a rolling wheel. Myc-Trap beads were magnetically

296 separated from the supernatant and washed 3 times in $500 \mu 1$

297 wash buffer. An aliquot corresponding to $10 \%$ of the beads

298 was used for immunoblotting and the remaining beads were

299 subjected to on-bead digestion with trypsin. For

300 immunoblotting of Co-IP samples, the proteins were eluted

301 from the beads in $30 \mu \mathrm{l}$ x SDS sample buffer $(250 \mathrm{mM}$ Tris-

$302 \mathrm{HCl} \mathrm{pH} \mathrm{6.8,} 40 \%$ glycerol, $8 \%$ SDS, $0.08 \%$ bromophenol

303 blue, $200 \mathrm{mM}$ DTT) by boiling for 10 minutes at $95^{\circ} \mathrm{C}$.

305 Depletion of free biotin. Biotin depletion methods were tested

306 using a transgenic line expressing miniTurbo-Myc-MpSYP13B

307 and Tak-1. Cell extracts were prepared as described above from 
308

309

310

311

312

313

314

315

316

317

318

319

320

321

322

323

324

325

326

327

328

329

330

331

332

333

334

335

336

337

338

339

340

10-day-old thalli treated with $700 \mu \mathrm{M}$ biotin for 24 hours. As input, $500 \mu \mathrm{g}$ of total protein in $500 \mu \mathrm{l}$ SDT buffer $(100 \mathrm{mM}$ Tris pH 7.5, 4 \% SDS, 0.1 M DTT) was used per sample. For methanol:chloroform precipitation, $666 \mu 1$ methanol and $166 \mu 1$ chloroform were added to the cell extracts and the samples were mixed. Next, $300 \mu 1$ water was further added and mixed, and then centrifuged for 10 minutes at 4,000 rpm. The separated upper and lower liquid phases were removed. The solid white layer containing precipitated proteins that had formed between the liquid phases was kept. The protein pellet was resuspended in $600 \mu \mathrm{l}$ methanol and sonicated for 10 minutes. After centrifugation for 10 minutes at 13,000 rpm, the supernatant was removed completely. The protein pellets were air-dried for 5 minutes and resuspended in $500 \mu$ SDT buffer. After 10 minutes sonication, the samples were incubated for 30 minutes at RT while shaking at 1,000 rpm, until the protein pellets were redissolved. The samples were then diluted with PBS buffer (0.1 M phosphate, $0.15 \mathrm{M} \mathrm{NaCl}, \mathrm{pH}$ 7.2) to a final concentration of $0.5 \%$ SDS. For biotin depletion with PD-10 desalting columns (VWR; 17085101), the columns were equilibrated with SDT:water (1:5) and the cell extracts were diluted to $2.5 \mathrm{ml}$ with ultrapure water. PD-10 desalting was performed according to the manufacturer's instructions and the proteins were eluted with $3.5 \mathrm{ml}$ PBS buffer containing $0.5 \%$ SDS. For biotin depletion using Zeba spin columns (Thermo; 89893), the columns were equilibrated with SDT:water (1:5) and the cell extracts were diluted to $2.5 \mathrm{ml}$ with ultrapure water. Desalting was then performed according to the manufacturer's instructions. All biotin-depleted samples were adjusted to $4 \mathrm{ml}$ final volume with binding buffer $(0.1 \mathrm{M}$ phosphate, $0.15 \mathrm{M}$ $\mathrm{NaCl}, 0.5 \%$ SDS, $\mathrm{pH}$ 7.2) before pulldown. Aliquots of intermediate steps were taken for immunoblotting. All biotin depletion methods were tested at the same time in duplicates. 
341 PD-10 column desalting method was employed for the pulled-

342 down samples measured by MS.

343

344 Pulldown of biotinylated proteins. Biotinylated proteins were

345 pulled-down using streptavidin-agarose beads (Thermo; 20353).

346 Per sample, $100 \mu 1$ of a $50 \%$ slurry were used. The beads were

347 washed and equilibrated in the binding buffer. Next, the biotin-

348 depleted samples were added to the beads and pulldown was

349 performed overnight at $22-25{ }^{\circ} \mathrm{C}$ while mixing. The beads

350 were washed once with $6 \mathrm{ml}$ PBS buffer containing $2 \%$ SDS

351 and then 3 times with $10 \mathrm{ml}$ PBS buffer. Aliquots of all

352 samples were taken during intermediate steps and an aliquot

353 corresponding to $10 \%$ of the washed beads was taken after

354 pulldown for immunoblotting. The proteins were eluted from

355 the beads by boiling for 10 minutes at $95^{\circ} \mathrm{C}$ in $50 \mu \mathrm{4x}$ SDS

356 sample buffer containing $20 \mathrm{mM}$ biotin while shaking at 1,000

357 rpm. For immunoblots, $20 \%$ of the IP-eluate was used,

358 corresponding to $2 \%$ of the input bead-amount. The remaining

359 beads from pulldown with bound biotinylated proteins were

360 subjected to on-bead digestion for MS analysis.

361

On-bead digestion. For Myc-IP and streptavidin-pulldown

363 samples, Myc-Trap beads or streptavidin-agarose beads were

364 resuspended in $25 \mu$ digest buffer 1 (50 mM Tris pH 7.5, $2 \mathrm{M}$

365 urea, $1 \mathrm{mM} \mathrm{DTT}, 5 \mu \mathrm{g} \mu \mathrm{l}^{-1}$ trypsin) and incubated at $30{ }^{\circ} \mathrm{C}$ for

36630 minutes while shaking at $400 \mathrm{rpm}$. The supernatant was

367 separated from the beads magnetically or by sedimentation and

368 transferred to a fresh tube. The beads were then mixed with 50

$369 \mu$ ligest buffer 2 (50 mM Tris pH 7.5, 2 M Urea, 5 mM CAA),

370 and the supernatant was separated from the beads and

371 combined with the supernatant from the previous step. The

372 combined supernatant was further incubated overnight at $32{ }^{\circ} \mathrm{C}$

373 while shaking at $400 \mathrm{rpm}$. Trypsin was inactivated by

374 acidification with trifluoroacetic acid (TFA), and the peptide 
375 sample was subsequently desalted using $\mathrm{C}_{18}$ stage tips

376 (Rappsilber et al. (2003).

377

Sample preparation for whole proteome analyses. Aliquots

of cell extracts from biotin-labelling experiments were used for

whole proteome analysis. The extracts were processed using a

filter-aided sample preparation (FASP) protocol adapted from

Wisnewski et al. (2009). In brief, $50 \mu \mathrm{g}$ of total protein extract

were used as input. The proteins were alkylated using chloroacetamide and digested using LysC and trypsin. The peptide solutions were desalted using $\mathrm{C}_{18}$ stage tips. Whole proteome analyses were conducted for one representative replicate per genotype and condition.

LC-MS/MS data acquisition. The dried peptides from filteraided digestion were re-dissolved in buffer $\mathrm{A}$ ( $2 \% \mathrm{ACN}, 0.1 \%$ TFA) and adjusted to a final peptide concentration of $0.1 \mu \mathrm{g} \mu \mathrm{l}^{-}$ Trap pulldowns were dissolved in $10 \mu \mathrm{l}$ buffer A and measured without dilution.

PL samples were analysed using an EASY-nLC 1200 (Thermo Fisher) coupled to a Q Exactive Plus mass spectrometer (Thermo Fisher). The peptides were separated on $16 \mathrm{~cm}$ frit-less silica emitters (New Objective, $75 \mu \mathrm{m}$ inner diameter), packed in-house with reversed-phase ReproSil-Pur

400 C18 AQ $1.9 \mu \mathrm{m}$ resin (Dr. Maisch). The peptides were loaded 401 on the column and eluted for 50 minutes using a segmented linear gradient of $5 \%$ to $95 \%$ solvent B (0 minutes: $5 \% \mathrm{~B}$; 0-5 minutes $\rightarrow 5 \% \mathrm{~B} ; 5-25$ minutes $\rightarrow 20 \% \mathrm{~B} ; 25-35$ minutes -> $35 \% \mathrm{~B} ; 35-40$ minutes $\rightarrow 95 \% \mathrm{~B}$; 40-50 minutes $>95 \%$ B) (solvent A: $0 \%$ ACN, $0.1 \% \mathrm{FA}$; solvent B: $80 \%$ ACN, $0.1 \%$ FA) at a flow rate of $300 \mathrm{nl}$ per minute. Mass spectra were acquired in data-dependent acquisition mode with 408 a TOP10 method. MS spectra were acquired in the Orbitrap 
409

410

411

412

413

414

415

416

417

418

419

420

421

422

423

424

425

426

427

428

429

430

431

432

433

434

435

436

437

438

439

440

441

442

analyser with a mass range of $300-1500 \mathrm{~m} / \mathrm{z}$ at a resolution of 70,000 FWHM and a target value of $3 \times 10^{6}$ ions. Precursors were selected with an isolation window of $1.3 \mathrm{~m} / \mathrm{z}$. HCD fragmentation was performed at a normalized collision energy of 25. MS/MS spectra were acquired with a target value of $5 \times 10^{5}$ ions at a resolution of 17,500 FWHM, a maximum injection time of 85 milliseconds and a fixed first mass of $\mathrm{m} / \mathrm{z}$ 100. Peptides with a charge of 1 , greater than 6 , or with unassigned charge state were excluded from fragmentation for $\mathrm{MS}^{2}$, dynamic exclusion for 20 seconds prevented repeated selection of precursors.

Myc-IP and whole proteome samples were analysed using an EASY-nLC 1000 (Thermo Fisher) coupled to a Q Exactive mass spectrometer (Thermo Fisher). The peptides were separated on $16 \mathrm{~cm}$ frit-less silica emitters (New Objective, $75 \mu \mathrm{m}$ inner diameter), packed in-house with reversed-phase ReproSil-Pur C18 AQ $1.9 \mu \mathrm{m}$ resin (Dr. Maisch). Peptides $(0.5 \mu \mathrm{g})$ were loaded on the column and eluted for 115 minutes using a segmented linear gradient of 5 $\%$ to $95 \%$ solvent $\mathrm{B}(0$ minutes: $5 \% \mathrm{~B}$; $0-5$ minutes $->5 \% \mathrm{~B}$; 5-65 minutes -> $20 \%$ B; 65-90 minutes -> 35\% B; 90-100 minutes $\rightarrow 55 \%$ B; 100-105 minutes $\rightarrow 95 \%$ B, 105-115 minutes $\rightarrow 95 \%$ B) (solvent A: $0 \% \mathrm{ACN}, 0.1 \% \mathrm{FA}$; solvent B: $80 \% \mathrm{ACN}, 0.1 \% \mathrm{FA})$ at a flow rate of $300 \mathrm{nl}$ per minute. Mass spectra were acquired in data-dependent acquisition mode with a TOP15 method. MS spectra were acquired in the Orbitrap analyser with a mass range of $300-1750 \mathrm{~m} / \mathrm{z}$ at a resolution of 70,000 FWHM and a target value of $3 \times 10^{6}$ ions. Precursors were selected with an isolation window of $2.0 \mathrm{~m} / \mathrm{z}$. HCD fragmentation was performed at a normalized collision energy of 25. MS/MS spectra were acquired with a target value of $10^{5}$ ions at a resolution of 17,500 FWHM, a maximum injection time of 55 milliseconds and a fixed first mass of $\mathrm{m} / \mathrm{z}$ 100. Peptides with a charge of 1 , greater than 6 , or with 
443 unassigned charge state were excluded from fragmentation for

$444 \mathrm{MS}^{2}$, dynamic exclusion for 30 seconds prevented repeated

445 selection of precursors.

446

447 Data analysis. Raw data were processed using MaxQuant

448 software (version 1.6.3.4, http://www.maxquant.org/) (Cox \&

449 Mann, 2008) with label-free quantification (LFQ) and intensity

450 based absolute quantification (iBAQ) enabled (Tyanova et al.,

451 2016). For PL data, normalization was skipped for the LFQ

452 quantification. MS/MS spectra were searched by the

453 Andromeda search engine against a combined database

454 containing the sequences from $M$. polymorpha

455 (MpTak1v5.1_r1_primary_transcripts_proteinV2;

456 http://marchantia.info/, (Montgomery et al., 2020)) and

457 sequences of 248 common contaminant proteins and decoy

458 sequences and the sequence of miniTurbo. Trypsin specificity

459 was required and a maximum of 2 missed cleavages allowed.

460 Minimal peptide length was set to 7 amino acids.

461 Carbamidomethylation of cysteine residues was set as fixed,

462 oxidation of methionine and protein $\mathrm{N}$-terminal acetylation as

463 variable modifications. Peptide-spectrum-matches and proteins

464 were retained if below a false discovery rate (FDR) of $1 \%$. For

465 PL data, the non-normalized MaxLFQ values of all replicates

466 (4 per condition) were pre-processed in Perseus (version 1.5.8.5,

467 http://www.maxquant.org/) and submitted for normalization

468 analysis using the Normalyzer tool

469 (http://normalyzer.immunoprot.lth.se/, (Chawade et al., 2014)).

470 The output was analysed for outliers and 1 replicate per

471 condition was removed. The final data analysis was conducted

472 in MaxQuant as described above on the reduced raw dataset.

473 Statistical analysis of the MaxLFQ values was conducted using

474 Perseus (version 1.5.8.5, http://www.maxquant.org/).

475 Quantified proteins were filtered for reverse hits and hits

476 "identified by site" and MaxLFQ values were $\log 2$ transformed. 
477 For PL data, transformed MaxLFQ values were normalized by

478 subtraction of the median per column. After grouping samples

479 by condition only those proteins were retained for subsequent

480 analysis that had 3 or 2 valid values in one of the conditions for

481 PL data or Myc-IP data, respectively. Two-sample $t$-tests were

482 performed using a permutation-based FDR of $5 \%$. For the

483 generation of volcano plots, missing values were imputed from

484 a normal distribution using the default settings in Perseus (1.8

485 downshift, separately for each column) for data with 3 valid

486 values in one of the conditions. Volcano plots were generated

487 in Perseus using an FDR of $5 \%$ and an $S O=1$. The Perseus

488 output was exported and further processed using Microsoft

489 Excel and RStudio (version 1.4.1103,

490 https://www.rstudio.com/), based on $\mathrm{R}$ (version x64 4.0.3,

491 https://cran.r-project.org/). The data was processed in RStudio

492 using tidyverse (version 1.3.0), rio (version 0.5.16) and zoo

493 (version 1.8-8) packages. Relative iBAQ values were

494 calculated per column from MaxQuant output, scaled by factor

$49510^{6}$ and $\log 10$ transformed. The median value of 3 replicates

496 per condition was used to generate volcano plots including

497 relative iBAQ values. Volcano pots were generated in RStudio

498 using the ggplot2 (version 3.3.3), ggrepel (version 0.9.1) and

499 ggsci (version 2.9) packages.

500

501 Immunoblotting. Proteins were separated by SDS-

502 polyacrylamide-gel electrophoresis (PAGE) and blotted onto

503 PVDF membranes (BioRad; 1704272) using a Trans-Blot

504 Turbo (BioRad) transfer system. The following antibodies were

505 used: streptavidin-HRP (Cell Signaling; 3999S), anti-Myc-tag

506 mouse monoclonal antibody (Cell Signaling; 9B11), HRP-

507 conjugated anti-mouse IgG antibody (Cell Signaling; 7076S).

508 The membranes were probed for biotinylated proteins with

509 streptavidin-HRP for 45 minutes to 3 hours at RT. For

510 detection of miniTurbo-Myc, the membranes were probed with 
511 anti-Myc-tag primary antibody overnight at $4{ }^{\circ} \mathrm{C}$ and then with

512 anti-mouse IgG secondary antibody for 1 hour at RT.

513 Biotinylated proteins or Myc-tagged miniTurbo were visualized

514 on the membranes using a luminol-based chemiluminescent

515 substrate that is oxidized by HRP in the presence of peroxide

516 (Thermo Fisher; 34577). The membranes were stained with

517 Coomassie staining solution $\left(60 \mathrm{mg} \mathrm{l}^{-1}\right.$ Coomassie brilliant blue,

$51810 \%$ acetic acid) afterwards.

519

520 Annotations and gene ontology analyses. For M. polymorpha

521 protein annotation, gene annotations from MpTak1_v5.1 and

522 JGI 3.1 (https://marchantia.info/) were integrated. Information

523 of A. thaliana homologs was further used for the annotation. A

524 Basic Local Alignment Search Tool (BLAST) was used to

525 determine homologs in A. thaliana (TAIR10). The best hit with

526 an e-value $\leq 10^{-10}$ was defined as a homolog. TAIR10

527 (https://www.arabidopsis.org/, (Lamesch et al., 2012)),

528 PANTHER version 16.0 (http://pantherdb.org/, (Mi et al.,

529 2021)), STRING 11.5 (https://string-db.org/, (von Mering et

530 al., 2003)), BioGrid 4.4 (https://thebiogrid.org/, (Stark et al.,

531 2006)), and IntAct 1.0.2 (https://www.ebi.ac.uk/intact/home,

532 (Orchard et al., 2014)) were used for annotating A. thaliana

533 homologs (Data S1). RStudio (version 1.4.1103,

534 https://www.rstudio.com/), based on R (version x64 4.0.3,

535 https://cran.r-project.org/), the tidyverse (version 1.3.0), rio

536 (version 0.5.16), and zoo (version 1.8-8) packages, were used

537 for integrating annotation files. GO-term enrichment

538 analysis was performed with Metascape 3.5

539 (https://metascape.org/, (Zhou et al., 2019)) using express

540 analysis settings. Corresponding A. thaliana homologs were

541 used as input protein lists for the analysis. A list of reported and

542 predicted interactors of AtSYP1 proteins was generated by

543 integrating information from BioGrid, IntAct, and STRING

544 databases (Data S2). BLAST was used to determine homologs 
545 in M. polymorpha (JGI 3.1). The best hit with an e-value $\leq 10^{-}$

$546{ }^{10}$ were defined as a homolog (Data S3). PANTHER version

54716.0 and PPDB (http://ppdb.tc.cornell.edu/, (Sun et al., 2009))

548 were used to predict plasma membrane-localization of $M$.

549 polymorpha homologs.

550 


\section{$551 \quad$ Results}

552 Experimental design for interactomics using miniTurbo553 mediated PL and Co-IP in M. polymorpha

554 To potentially enhance transcription and translation efficiency

555 of the bait-ligase fusion-proteins in M. polymorpha, we used a

556 codon-optimized version of the original miniTurbo (Fig. S1).

557 We added a single Myc-tag to the C-terminus of miniTurbo, 558 enabling not only the detection of the miniTurbo fusion-

559 proteins by immunoblotting, but also Co-IP experiments (Fig.

560 1a, Fig. S1). We designed binary vectors to express miniTurbo-

561 Myc-MpSYP12A and miniTurbo-Myc-MpSYP13B fusion-

562 proteins under the native promoters of $\mathrm{MpSYP} 12 \mathrm{~A}$ and

563 MpSYP13B genes, respectively (Fig. 1a, Fig. S2). Using these

564 constructs, we generated stable transgenic lines in wildtype

565 Tak-1 background. Expression of the miniTurbo-Myc fusion-

566 proteins in candidate transformant lines was confirmed by

567 immunoblotting using an anti-Myc antibody. To establish and

568 evaluate the miniTurbo-based interactomics method, we

569 selected transgenic lines showing similar expression levels for

570 miniTurbo-Myc-MpSYP12A and miniTurbo-Myc-MpSYP13B.

571 For this study, we used line No. 1 and line No. 3 for

572 miniTurbo-Myc-MpSYP12A and miniTurbo-Myc-MpSYP13B,

573 respectively (Fig. S3a).

574 In A. thaliana, Mair et al. (2019) demonstrated that

575 levels of miniTurbo-mediated biotinylation of cellular proteins

576 saturated when plants were treated with $50 \mu \mathrm{M}$ biotin solution.

577 Since uptake of exogenous biotin and levels of endogenously

578 produced biotin are unknown in M. polymorpha, we treated the

579 selected transgenic lines and Tak-1 with different

580 concentrations of biotin, $0-700 \mu \mathrm{M}$, to find suitable conditions

581 for in vivo biotin-labelling. For biotin treatment, thalli were

582 grown from single gemmae for 10 days, and then whole plants

583 were submerged in biotin solutions and vacuum infiltrated for 5 
584 minutes. We incubated the thalli in biotin solutions at $22-25^{\circ} \mathrm{C}$

585 for 24 hours, a time point at which we expected a saturation of

586 biotin-labelling based on previous studies using miniTurbo in

587 other plant species (Mair et al., 2019; Zhang et al., 2019). We

588 subsequently checked the levels of biotinylated proteins in cell

589 extracts by immunoblotting using streptavidin-HRP (Fig. 1c,

590 Fig. S4a). We found increasing levels of biotinylated proteins

591 with increasing biotin concentration in cell extracts of

592 transgenic lines expressing either miniTurbo-Myc-MpSYP12A

593 or miniTurbo-Myc-MpSYP13B but not in Tak-1. This

594 confirmed biotin uptake and miniTurbo biotin ligase activity in

595 M. polymorpha under the tested conditions. Given that levels of

596 biotinylated proteins did not saturate for biotin concentrations

597 up to $700 \mu \mathrm{M}$ in $M$. polymorpha, we used $700 \mu \mathrm{M}$ biotin

598 solution in all following experiments. To determine suitable

599 biotin treatment times in M. polymorpha, we next performed a

600 time-course experiment and checked the levels of biotinylated

601 proteins by immunoblotting after 0-24 hours of biotin

602 treatment (Fig. 1d). We detected increased levels of

603 biotinylated proteins in samples of transgenic lines expressing

604 either miniTurbo-Myc-MpSYP12A or miniTurbo-Myc-

605 MpSYP13B after 30 minutes of treatment (Fig. S4b), which

606 further increased over time up to 24 hours of treatment (Fig.

607 1d).

608 Previously published studies using TurboID and 609 miniTurbo identified the depletion of free biotin after labelling 610 as a critical step for pulldown of biotinylated proteins using 611 streptavidin beads (Mair et al., 2019; Zhang et al., 2019; Arora 612 et al., 2020; Zhang et al., 2021). We therefore tested three 613 different approaches, methanol:chloroform precipitation, PD-10 614 gravity column desalting, and Zeba spin column desalting, to 615 remove excess free biotin from $M$. polymorpha cell extracts. 616 We used the miniTurbo-Myc-MpSYP13B line, which was 617 treated with $700 \mu \mathrm{M}$ biotin for 24 hours. We evaluated biotin 
618 depletion based on pulldown efficiency, through 619 immunoblotting of biotinylated proteins that could be eluted 620 from the streptavidin-agarose beads after pulldown, and of non-

621 bound biotinylated proteins that remained in the supernatant of 622 the beads (Fig. S3b, c). We found that all three methods could 623 be used before affinity-pulldown to sufficiently enrich 624 biotinylated proteins from M. polymorpha samples (Fig. S3b). 625 With respect to easier handling, we used PD-10 column 626 desalting for subsequent experiments.

627

The miniTurbo-based approach identifies a manifold of potential interactors of MpSYP12A and MpSYP13B in comparison to the Co-IP approach

631 A direct comparison between the performances of PL and Co-

632 IP approaches for interactomics using the same plant materials 633 has not yet been reported. We therefore performed IP of 634 miniTurbo-Myc-MpSYP12A or miniTurbo-Myc-MpSYP13B 635 using Myc-Trap beads from the same selected transgenic lines. 636 After IP, we checked successful pulldown of the miniTurbo637 Myc fusion-proteins by immunoblotting. We detected 638 miniTurbo-Myc-MpSYP12A and miniTurbo-Myc-MpSYP13B 639 fusion-proteins in cell extract that was used as input for IP, and 640 in IP-eluates of samples from the transgenic lines (Fig. S3d). 641 We then identified and quantified the proteins captured by 642 Myc-IP using MS. We found a significant enrichment of the 643 two bait proteins (Fig. 2a), confirming the immunoblotting 644 result. Using Co-IP, we identified 4 and 1 potential interactors of MpSYP12A and MpSYP13B, respectively (Fig. 2a).

For the PL approach, we treated plants with biotin for 4 647 and 24 hours to identify and quantify proteins after pulldown 648 by MS. As in the case of the Co-IP approach, we found a 649 significant enrichment of both baits using the miniTurbo-based 650 approach (Fig. 2b, c). Four hours of biotin treatment resulted in 651 an identification of 214 and 189 proteins as potential interactors 
652 of MpSYP12A and MpSYP13B, respectively (Fig. 2b). By

653 increasing the treatment time to 24 hours, the numbers of

654 identified potential interactors nearly tripled (Fig. 2c). As

655 expected, most of the candidates identified from 4 hours'

656 samples were also identified from 24 hours' samples (Fig. 2d).

657 In other words, approximately one third of the candidates that

658 were identified after 24 hours of biotin treatment could be

659 identified after 4 hours of treatment. MpCSR1

660 (CHLORSULFURON RESISTANT 1), a potential interactor of

661 MpSYP12A and MpSYP13B that we identified using Co-IP,

662 was identified in the 24 hours' PL interactome dataset as well

663 (Fig. 2c). Overall, these results demonstrate that PL approaches

664 have a higher potential to identify undescribed interacting

665 proteins compared to Co-IP approaches. However, it should be

666 noted that the miniTurbo-based approach failed to identify

667 proteins like secretory peroxidases that are predicted to be

668 secreted into the extracellular space (Fig. 2a). This is

669 reasonable as miniTurbo was fused to the intracellular domain

670 of MpSYPs.

671

PL using MpSYP12A and MpSYP13B as baits enriches proteins involved in vesicle-mediated transport and plasma membrane-localized proteins

675 We next asked whether the potential interactors that we 676 identified by PL overall fit to the expected biological function 677 of MpSYP12A and MpSYP13B. For this, we annotated $M$. 678 polymorpha proteins based on information of $A$. thaliana 679 homologs (Data S1). We then performed gene ontology (GO)680 term enrichment analysis of the 24 hours interactome dataset 681 using Metascape. Strikingly, 'vesicle-mediated transport' was 682 the most significantly enriched GO-term extracted from the 683 interactome data for both, MpSYP12A and MpSYP13B, 684 coinciding with SNARE protein functions (Fig. 3a, b; Data S4). 685 We also performed GO-term enrichment analysis of proteome 
686 data that were obtained by measuring the input samples used

687 for the streptavidin-pulldown. Enriched GO-terms from

688 proteome data were mainly related to primary metabolism (Fig.

$6893 \mathrm{c})$, which is clearly distinct from the enriched GO-terms of the

690 interactome dataset (Fig. 3a, b). These results suggest that the

691 potential interactors comprise actual interactors of MpSYP12A

692 and MpSYP13B. Analysis of the 4 hours interactome dataset

693 gave similar results (Fig. S5a, b; Data S4).

694 A number of interactors of $A$. thaliana SNAREs, which

695 are homologous to MpSYP12A and MpSYP13B, have been

696 reported (Kwon et al., 2008; Fujiwara et al., 2014). Based on

697 BioGrid, STRING, and IntAct databases, a total of 334 proteins

698 were reported or predicted to interact with SYP1 proteins in A.

699 thaliana (Data S2). Of these 334 A. thaliana proteins, we could

700 identify 250 homologous proteins conserved in the $M$.

701 polymorpha proteome using a BLAST approach (Data S3).

702 Among these 250 proteins, we found that 47 and 40 proteins

703 were identified as potential interactors of MpSYP12A and

704 MpSYP13B, while 39 were shared between both baits,

705 respectively. This means that around 11-15\% of all potential

706 interactors of MpSYP12A and MpSYP13B revealed by the

707 miniTurbo-based approach are homologous to known

708 interactors of A. thaliana SYP1 proteins (Fig. 3d).

709 MpSYP12A and MpSYP13B were demonstrated to

710 localize to the plasma membrane and to be ubiquitously

711 expressed throughout the thallus in M. polymorpha (Kanazawa

712 et al., 2016). By using PANTHER GO annotations and A.

713 thaliana plasma membrane proteome data, we predicted plasma

714 membrane localizations of the potential interactors in $M$.

715 polymorpha (Data S1). We found that more than one third of

716 the potential interactors are expected to be plasma membrane-

717 localized (Fig. 3e). This result further supported the specificity

718 and utility of the miniTurbo-based approach. 


\section{The miniTurbo-mediated approach can reveal subtle}

\section{1 differences between very similar baits}

722 By comparing the 24 hours interactome data for MpSYP12A

723 and MpSYP13B, we found that both baits share 90-95\% of

724 their potential interactors (Fig. 1c), which is reasonable

725 considering their predicted functions. Still, we captured 52 and

7269 proteins that preferentially interact with MpSYP12A and

727 MpSYP13B, respectively (Fig. 4a, Table 1). To investigate

728 exclusive interaction partners of MpSYP12A and MpSYP13B,

729 we implemented iBAQ (Intensity Based Absolute

730 Quantification) values to the volcano plot (Fig. 4b). The iBAQ

731 is a measure of protein abundance, and relative abundance was

732 reflected in different circle sizes. The respective colours

733 indicate abundances in all pulldown samples. By this, we

734 revealed that MpNEK potentially interacts exclusively with

$735 \mathrm{MpSYP13B}$, and we found 13 proteins that potentially interact

736 exclusively with MpSYP12A (Fig. 4b, Table 1). 


\section{Discussion}

739 Published studies applying in planta TurboID-based PL have 740 utilized model angiosperm species, and the amenability of 741 current PL approaches to other plant species remains to be 742 determined as outlined by Mair and Bergmann (2021). Here, 743 we successfully applied miniTurbo-based PL in the liverwort $744 M$. polymorpha. This confirmed the transferability of biotin 745 ligase utilized interactomics to a model bryophyte species.

$746 \quad$ We tested different biotin concentrations and treatment 747 times to investigate biotin-labelling of proteins in $M$. 748 polymorpha cells. Mair et al. (2019) reported a saturation of 749 biotinylated proteins in A. thaliana stable transgenic lines after 750 treatment with $50 \mu \mathrm{M}$ biotin solution for 1 hour. Zhang et al. 751 (2019) transiently expressed TurboID-fusion-proteins in $N$. 752 benthamiana leaves and tested biotin concentrations up to 800 $753 \mu \mathrm{M}$. Zhang et al. observed that protein biotinylation was 754 saturated after 8 hours of treatment with $200 \mu \mathrm{M}$ biotin, and 755 that 15 minutes were sufficient for the saturation at $200 \mu \mathrm{M}$ 756 biotin concentration. Meanwhile, in M. polymorpha, we did not 757 observe a saturation of protein biotinylation after 24 hours of 758 treatment with $700 \mu \mathrm{M}$ biotin. The observed differences in 759 saturation of biotinylated proteins among the different studies 760 could be partially explained by differences in mechanisms and 761 efficiencies for biotin uptake and metabolism. Other factors 762 potentially impacting biotin-labelling activities are plant growth 763 conditions like light cycle and temperature used during biotin 764 treatment.

765 For PL approaches, a careful evaluation of false positive 766 candidates based on well-designed controls is desirable to 767 generate a set of candidates with high confidence for further 768 analyses and validation. It would be beneficial to include other 769 plasma membrane-localized proteins that are independent of 770 MpSYP12A and MpSYP13B to aid in predicting false positive 
771 candidates, which might have been biotinylated randomly due

772 to their localizations. That is to say, our transgenic lines

773 expressing miniTurbo-Myc-MpSYP12A and miniTurbo-Myc-

774 MpSYP13B could be used as suitable controls for interactome

775 mapping using other plasma membrane-localized baits in the

776 future. Meanwhile, the overall high similarity between

777 MpSYP12A and MpSYP13B can be exploited to investigate

778 specific interactors to understand functional differences

779 between the two SNAREs. In general, long biotin treatment

780 times potentially increase false positive labelling. On the other

781 hand, longer treatment times may be required to efficiently

782 capture rare or transient interactors.

783 For biotin-labelling approaches, protein extraction can

784 be conducted in the presence of strong detergents. Strong

785 detergents facilitate the extraction and solubilization of

786 membrane-associated proteins, which can be advantageous for

787 interactomics of plasma membrane-localized or organellar

788 proteins. Our results confirmed that we could indeed capture a

789 manifold of proteins that are predicted to be plasma membrane-

790 localized, which has not been tested on the proteome level in

791 published studies using PL in plants. In contrast to the Co-IP

792 approach, the miniTurbo-based method could not capture

793 extracellular interactors of MpSYP12A and MpSYP13B, which

794 can be a drawback for certain applications. In the future, it

795 remains to be determined whether the miniTurbo-based

796 approach is also suitable for other cellular compartments,

797 which may have a different intraorganellar $\mathrm{pH}$ or temperature,

798 as discussed by Mair and Bergmann (2021).

799 Until to date, a manifold of interaction partners of

800 AtSYP1 family proteins has been identified or predicted (Kwon

801 et al., 2008; Arabidopsis Interactome Mapping Consortium,

802 2011; Fujiwara et al., 2014);. With our miniTurbo-based

803 approach, we were able to capture homologs to well-known

804 AtSYP1-interacting proteins, such as KEU, NPSN11, SYP61, 
805 and VAMP721 (Fujiwara et al., 2014), demonstrating the

806 reliability of our method. We identified 47 and 40 proteins that

807 are homologous to previously described AtSYP1-interacting

808 proteins, while 104 and 92 proteins were linked to 'vesicle-

809 mediated transport' based on our GO-term enrichment analysis.

810 This indicates that we were able to capture a number of

811 undescribed potential interactors of MpSYP12A and

812 MpSYP13B, respectively. Kanazawa et al. (2020) were able to

813 show that MpSYP12A but not MpSYP13B localized to the

814 phragmoplast during cell plate formation by using fluorescent

815 reporter-lines. Based on our GO-term enrichment analysis, we

816 found the same set of 30 proteins related to 'cell plate

817 formation' in interactomes of both, MpSYP12A and

818 MpSYP13B. In other words, we failed to identify unique

819 potential interactors of MpSYP12A previously linked to 'cell

820 plate formation'. To address a role of MpSYP12A at the

821 phragmoplast, experimental conditions would need to be

822 further adjusted to capture the interactome during cell division,

823 by using cell cycle specific promoters to express proteins in

824 dividing cells, for instance.

825 Our miniTurbo-based approach identified a number of 826 potential interactors that are specific for MpSYP12A and 827 MpSYP13B, which may help to understand functional 828 differences between the two SNAREs in the future. For 829 example, we found that homologs to MILDEW RESISTANCE 830 LOCUS O (MLO) and PENETRATION 3 (PEN3) 831 preferentially interact with MpSYP12A. MLO genes were 832 shown to be involved in susceptibility to powdery mildew 833 pathogens in barley (Büschges et al., 1997) and A. thaliana 834 (Consonni et al., 2006). AtPEN3 was found to be involved in 835 resistance against barley powdery mildew (Stein et al., 2006) 836 and cell-death responses upon infection with $P$. infestans 837 (Kobae et al., 2006). In A. thaliana, AtSYP121 or 838 PENETRATION1 (PEN1) is homologous to MpSYP12A. 
839 AtPEN1 was shown to be required for SNARE-dependent

840 penetration resistance against barley powdery mildew and

841 pathogen-induced vesicle accumulation was enhanced in $M L O$

842 loss-of-function mutants (Collins et al., 2003). Recently,

843 Rubiato et al. (Rubiato et al., 2021) provided evidence for an

844 evolutionary conserved role of SYP12 proteins in the formation

845 of papillae and encasements at pathogen penetration sites,

846 which are effective defence structures against a broad range of

847 filamentous pathogens. Taken together, our data suggest a

848 function of MpSYP12A in penetration resistance and responses

849 to filamentous pathogens, like SYP12 proteins in other plant

850 species. Besides, we found that MpSYP12A may exclusively

851 interact with ABSCISIC ACID INSENSITIVE1 (MpABI1),

852 NUCLEAR MATRIX CONSTITUENT PROTEIN

853 (MpNMCP), and homologs of OROSOMUCOID-LIKE 1

854 (ORM1) and SENSITIVE TO FREEZING 2 (SFR2). MpABI1

855 is involved in abscisic acid signalling (Tougane et al., 2010)

856 and MpNMCP was found to function in stress signalling in $M$.

857 polymorpha (Wang et al., 2021). In A. thaliana, NMCP

858 homologs play a role in immunity (Choi et al., 2019; Jarad et

859 al., 2019). AtORMs were reported to play roles in sphingolipid

860 homeostasis and stress responses (Li et al., 2016), and AtSFR2

861 is a membrane remodelling enzyme responsive to freezing

862 conditions in A. thaliana (Barnes et al., 2019). Accordingly,

863 our findings may suggest a potential role of MpSYP12A in

864 lipid homeostasis and stress responses. We identified NIMA-

865 related protein kinase 1 (MpNEK) as potential exclusive

866 interactor for MpSYP13B. MpNEK directs tip growth in

867 rhizoids of $M$. polymorpha (Otani et al., 2018), and thus our

868 data may suggest a potential role of MpSYP13B in rhizoid tip

869 growth. It should be noted that potential interactors revealed by

870 PL need to be validated using complementary approaches. In

871 summary, our interactome data should be a useful resource for 
bioRxiv preprint doi: https://doi.org/10.1101/2022.01.21.477208; this version posted January 25, 2022. The copyright holder for this preprint (which was not certified by peer review) is the author/funder. All rights reserved. No reuse allowed without permission.

872 future investigations of functional conservation and

873 diversification of SNARE proteins in plants.

874

875 


\section{Acknowledgements}

877 We thank Takashi Ueda (National Institutes of Natural

878 Sciences, Division of Cellular Dynamics, Tokyo, Japan) for

879 providing the plasmids harbouring genomic sequences for

880 native expression and regulation of MpSYP12A and

881 MpSYP13B. We thank Takayuki Kohchi (Kyoto University,

882 Japan) for providing pMpGWB vectors.

883

884 Author Contribution

$885 \mathrm{KM}$ and $\mathrm{HN}$ have designed the research. KM, SCS, AH, and

$886 \mathrm{HN}$ have contributed to experimental design and workflow

887 conceptualization. KM, SCS, and AH have conducted

888 experiments. KM, SCS, and HN have analysed the data by

889 mass spectrometry. KM, SCS, and HN wrote the manuscript.

890 This project was supported by the Max Planck Society and was

891 conducted in the framework of MAdLand

892 (http://madland.science, Deutsche Forschungsgemeinschaft

893 (DFG) priority program 2237). HN is grateful for funding by

894 the DFG (NA 946/1-1).

895

896 Data Availability

897 The mass spectrometry proteomics data have been deposited to

898 the ProteomeXchange Consortium via the PRIDE [1] partner

899 repository with the dataset identifier PXD030429.

900 
901

902

903

904

905

906

907

908

909

910

911

912

913

914

915

916

917

918

919

920

921

922

923

924

925

926

927

928

929

930

931

932

933

934

935

936

937

938

939

940

941

942

943

944

945

946

947

948

949

\section{References}

Arora D, Abel NB, Liu C, Van Damme P, Yperman K, Eeckhout D, Vu LD, Wang J, Tornkvist A, Impens F, et al. 2020. Establishment of Proximity-Dependent Biotinylation Approaches in Different Plant Model Systems. Plant Cell 32(11): 3388-3407.

Baldet P, Alban C, Douce R. 1997. Biotin synthesis in higher plants: purification and characterization ofbioBgene product equivalent fromArabidopsis thalianaoverexpressed inEscherichia coliand its subcellular localization in pea leaf cells. FEBS Letters 419(2-3): 206-210.

Baldet P, Gerbling H, Axiotis S, Douce R. 1993. Biotin biosynthesis in higher plant cells. Eur. J. Biochem. 217: 479-485.

Barnes AC, Elowsky CG, Roston RL. 2019. An Arabidopsis protoplast isolation method reduces cytosolic acidification and activation of the chloroplast stress sensor SENSITIVE TO FREEZING 2. Plant Signal Behav 14(9): 1629270.

Batoko H, Moore I. 2001. Plant cytokinesis: KNOLLE joins the club. Curr Biol 11: R423-R426.

Bowman JL, Kohchi T, Yamato KT, Jenkins J, Shu S, Ishizaki K, Yamaoka S, Nishihama R, Nakamura Y, Berger F, et al. 2017. Insights into Land Plant Evolution Garnered from the Marchantia polymorpha Genome. Cell 171(2): 287-304 e215.

Branon TC, Bosch JA, Sanchez AD, Udeshi ND, Svinkina T, Carr SA, Feldman JL, Perrimon N, Ting AY. 2018. Efficient proximity labeling in living cells and organisms with TurbolD. Nat Biotechnol 36(9): 880-887.

Büschges R, Hollricher K, Panstruga R, Simons G, Wolter M, Frijters $A$, van Daelen $R$, van der Lee $T$, Diergaarde $P$, Groenendijk J, et al. 1997. The Barley Mlo Gene: A Novel Control Element of Plant Pathogen Resistance. Cell 88: 695-705.

Carella P, Gogleva A, Tomaselli M, Alfs C, Schornack S. 2018. Phytophthora palmivora establishes tissue-specific intracellular infection structures in the earliest divergent land plant lineage. Proc Natl Acad Sci U S A 115(16): E3846E3855.

Catalano CM, Czymmek KJ, Gann JG, Sherrier DJ. 2007. Medicago truncatula syntaxin SYP132 defines the symbiosome membrane and infection droplet membrane in root nodules. Planta 225(3): 541-550.

Chawade A, Alexandersson E, Levander F. 2014. Normalyzer: a tool for rapid evaluation of normalization methods for omics data sets. J Proteome Res 13(6): 3114-3120.

Choi J, Strickler SR, Richards EJ. 2019. Loss of CRWN Nuclear Proteins Induces Cell Death and Salicylic Acid Defense Signaling. Plant Physiol 179(4): 1315-1329.

Collins NC, Thordal-Christensen HVL, V., Bau S, Kombrink E, Qiu J-L, Hückelhoven R, Stein M, Freialdenhoven A, Somerville SC, 
Schulze-Lefert P. 2003. SNARE-protein-mediated disease resistance at the plant cell wall. Nature 425: 973-977.

Consonni C, Humphry ME, Hartmann HA, Livaja M, Durner J, Westphal L, Vogel J, Lipka V, Kemmerling B, Schulze-Lefert $P$, et al. 2006. Conserved requirement for a plant host cell protein in powdery mildew pathogenesis. Nat Genet 38(6): 716-720.

Consortium AIM. 2011. Evidence for Network Evolution in an Arabidopsis Interactome Map. Science 333: 601-607.

Cox J, Mann M. 2008. MaxQuant enables high peptide identification rates, individualized p.p.b.-range mass accuracies and proteome-wide protein quantification. Nat Biotechnol 26(12): 1367-1372.

Enami K, Ichikawa M, Uemura T, Kutsuna N, Hasezawa S, Nakagawa T, Nakano A, Sato MH. 2009. Differential expression control and polarized distribution of plasma membrane-resident SYP1 SNAREs in Arabidopsis thaliana. Plant Cell Physiol 50(2): 280-289.

Fujiwara M, Uemura T, Ebine K, Nishimori Y, Ueda T, Nakano A, Sato MH, Fukao Y. 2014. Interactomics of Qa-SNARE in Arabidopsis thaliana. Plant Cell Physiol 55(4): 781-789.

Hirano T, Ebine K, Ueda T, Higaki T, Nakayama T, Konno H, Takigawa-Imamura H, Sato MH. 2020. The SYP123VAMP727 SNARE complex is involved in the delivery of inner cell wall components to the root hair shank in Arabidopsis. biorxiv.

Ichikawa M, Hirano T, Enami K, Fuselier T, Kato N, Kwon C, Voigt B, Schulze-Lefert P, Baluska F, Sato MH. 2014. Syntaxin of plant proteins SYP123 and SYP132 mediate root hair tip growth in Arabidopsis thaliana. Plant Cell Physio/ 55(4): 790800.

Ishizaki K, Chiyoda S, Yamato KT, Kohchi T. 2008. Agrobacteriummediated transformation of the haploid liverwort Marchantia polymorpha L., an emerging model for plant biology. Plant Cell Physiol 49(7): 1084-1091.

Ishizaki K, Johzuka-Hisatomi Y, Ishida S, lida S, Kohchi T. 2013. Homologous recombination-mediated gene targeting in the liverwort Marchantia polymorpha L. Sci Rep 3: 1532.

Ishizaki K, Nishihama R, Ueda M, Inoue K, Ishida S, Nishimura Y, Shikanai T, Kohchi T. 2015. Development of Gateway Binary Vector Series with Four Different Selection Markers for the Liverwort Marchantia polymorpha. PLoS One 10(9): e0138876.

Jarad M, Mariappan K, Almeida-Trapp M, Mette MF, Mithofer A, Rayapuram N, Hirt H. 2019. The Lamin-Like LITTLE NUCLEI 1 (LINC1) Regulates Pattern-Triggered Immunity and Jasmonic Acid Signaling. Front Plant Sci 10: 1639.

Johansson ON, Fantozzi E, Fahlberg P, Nilsson AK, Buhot N, Tor M, Andersson MX. 2014. Role of the penetration-resistance genes PEN1, PEN2 and PEN3 in the hypersensitive response 
et al. 2020. Chromatin Organization in Early Land Plants Reveals an Ancestral Association between H3K27me3, Transposons, and Constitutive Heterochromatin. Curr Biol 30(4): 573-588 e577.

Orchard S, Ammari M, Aranda B, Breuza L, Briganti L, BroackesCarter F, Campbell NH, Chavali G, Chen C, del-Toro N, et al. 2014. The MIntAct project--IntAct as a common curation platform for 11 molecular interaction databases. Nucleic Acids Res 42(Database issue): D358-363.

Otani K, Ishizaki K, Nishihama R, Takatani S, Kohchi T, Takahashi T, Motose H. 2018. An evolutionarily conserved NIMA-related kinase directs rhizoid tip growth in the basal land plant Marchantia polymorpha. Development 145(5).

Pommerrenig B, Popko J, Heilmann M, Schulmeister S, Dietel K, Schmitt B, Stadler R, Feussner I, Sauer N. 2013. SUCROSE TRANSPORTER 5 supplies Arabidopsis embryos with biotin and affects triacylglycerol accumulation. Plant J 73(3): 392404.

Rappsilber J, Ishihama Y, Mann M. 2003. Stop and Go Extraction Tips for Matrix-Assisted Laser Desorption/Ionization, Nanoelectrospray, and LC/MS Sample Pretreatment in Proteomics. Anal Chem 75: 663-670.

Reichardt I, Slane D, El Kasmi F, Knoll C, Fuchs R, Mayer U, Lipka V, Jurgens G. 2011. Mechanisms of functional specificity among plasma-membrane syntaxins in Arabidopsis. Traffic 12(9): 1269-1280.

Rubiato HM, Liu M, O'Connell RJ, Nielsen ME. 2021. Plant SYP12 syntaxins mediate an evolutionarily conserved general immunity to filamentous pathogens. biorxiv.

Sanderfoot A. 2007. Increases in the number of SNARE genes parallels the rise of multicellularity among the green plants. Plant Physiol 144(1): 6-17.

Silva PA, UI-Rehman R, Rato C, Di Sansebastiano G-P, Malhó R. 2010. Asymmetric localization of Arabidopsis SYP124 syntaxin at the pollen tube apical and sub-apical zones is involved in tip growth. BMC Plant Biol 10(179): 1-12.

Stark C, Breitkreutz BJ, Reguly T, Boucher L, Breitkreutz A, Tyers M. 2006. BioGRID: a general repository for interaction datasets. Nucleic Acids Res 34(Database issue): D535-539.

Stein M, Dittgen J, Sanchez-Rodriguez C, Hou BH, Molina A, Schulze-Lefert P, Lipka V, Somerville S. 2006. Arabidopsis PEN3/PDR8, an ATP binding cassette transporter, contributes to nonhost resistance to inappropriate pathogens that enter by direct penetration. Plant Cell 18(3): 731-746.

Sun Q, Zybailov B, Majeran W, Friso G, Olinares PD, van Wijk KJ. 2009. PPDB, the Plant Proteomics Database at Cornell. Nucleic Acids Res 37(Database issue): D969-974.

Tougane K, Komatsu K, Bhyan SB, Sakata Y, Ishizaki K, Yamato KT, Kohchi T, Takezawa D. 2010. Evolutionarily conserved regulatory mechanisms of abscisic acid signaling in land 
plants: characterization of ABSCISIC ACID INSENSITIVE1-like type $2 C$ protein phosphatase in the liverwort Marchantia polymorpha. Plant Physiol 152(3): 1529-1543.

Tyanova S, Temu T, Cox J. 2016. The MaxQuant computational platform for mass spectrometry-based shotgun proteomics. Nat Protoc 11(12): 2301-2319.

Uemura T, Kim H, Saito C, Ebine K, Ueda T, Schulze-Lefert P, Nakano A. 2012. Qa-SNAREs localized to the trans-Golgi network regulate multiple transport pathways and extracellular disease resistance in plants. Proc Natl Acad Sci US A 109(5): 1784-1789.

Uemura T, Ueda T, Ohniwa RL, Nakano A, Takeyasu K, Sato MH. 2004. Systematic Analysis of SNARE Molecules in Arabidopsis: Dissection of the post-Golgi Network in Plant Cells. CELL STRUCTURE AND FUNCTION 29: 49-65.

von Mering C, Huynen M, Jaeggi D, Schmidt S, Bork P, Snel B. 2003. STRING: a database of predicted functional associations between proteins. Nucleic Acids Res 31(1): 258-261.

Wang N, Karaaslan ES, Faiss N, Berendzen KW, Liu C. 2021. Characterization of a Plant Nuclear Matrix Constituent Protein in Liverwort. Front Plant Sci 12: 670306.

Wisniewski JR, Zougman A, Nagaraj N, Mann M. 2009. Universal sample preparation method for proteome analysis. Nat Methods 6(5): 359-362.

Xia L, Mar Marques-Bueno M, Bruce CG, Karnik R. 2019. Unusual Roles of Secretory SNARE SYP132 in Plasma Membrane $\mathrm{H}(+)$ ATPase Traffic and Vegetative Plant Growth. Plant Physiol 180(2): 837-858.

Yun HS, Kang BG, Kwon C. 2016. Arabidopsis immune secretory pathways to powdery mildew fungi. Plant Signal Behav 11(10): e1226456.

Zhang Y, Li Y, Wen Z, Nagalakshmi U, Dinesh-Kumar SP. 2021. TurbolD-Based Proximity Labeling for In Planta Identification of Protein-Protein Interaction Networks. J Vis Exp: 1-15.

Zhang Y, Song G, Lal NK, Nagalakshmi U, Li Y, Zheng W, Huang PJ, Branon TC, Ting AY, Walley JW, et al. 2019. TurbolD-based proximity labeling reveals that UBR7 is a regulator of N NLR immune receptor-mediated immunity. Nat Commun 10(1): 3252.

Zhou Y, Zhou B, Pache L, Chang M, Khodabakhshi AH, Tanaseichuk O, Benner C, Chanda SK. 2019. Metascape provides a biologist-oriented resource for the analysis of systems-level datasets. Nat Commun 10(1): 1523. 


\section{$1148 \quad$ Figure legends}

1149

1150

Fig. 1 Experimental setup for miniTurbo-mediated biotin-labelling in

1151

M. polymorpha. (a) Schematic representation of the constructs used for

1152 generating transgenic plants. (b) Overview of the workflow used for evaluating the miniTurbo-based interactomics method in M. polymorpha.

The figure was created with elements from BioRender (https://biorender.com). (c and d) Biotin ligase activity in M. polymorpha. Streptavidin (SA) immunoblots (IB) of cell extracts from M. polymorpha transgenic lines expressing miniTurbo-Myc-MpSYP12A (upper panels) and miniTurbo-Myc-MpSYP13B (lower panels) that were (c) treated with 0-700 $\mu \mathrm{M}$ biotin solutions for 24 hours at RT or (d) treated with $700 \mu \mathrm{M}$ biotin solution for 0-24 hours at RT. Cell extracts of wildtype Tak-1 (WT) treated with $700 \mu \mathrm{M}$ biotin solution for 24 hours were used as a control. Arrows indicate the positions of the biotinylated miniTurbo-MycMpSYP12A and miniTurbo-Myc-MpSYP13B fusion-proteins. Coomassie Brilliant Blue-stained (CBB) membranes are shown as loading controls.

Fig. 2 Identification of MpSYP12A or MpSYP13B interacting proteins

by Co-IP and PL approaches. Wildtype Tak-1 was used as a control, and proteins that are significantly co-purified with or biotinylated by baits are highlighted. Potential interacting proteins for MpSYP12A and MpSYP13B are shown in turquoise and magenta, respectively. Venn diagrams show numbers of the potential interactors and their overlaps. (a) Myc-Trap Co-IP. Black text labels in volcano plots indicate the potential interactors for MpSYP12A or MpSYP13B. (b) 4 hours PL. (c) 24 hours PL. (d) Overlaps between 4 hours and 24 hours PL. Black circles in volcano plots indicate the potential interactors that are also identified with 4 hours PL. Dark grey circles indicate proteins that are identified as the potential interactors with 4 hours PL but not with 24 hours PL.

Fig. 3 Features of the identified MpSYP12A or MpSYP13B interacting proteins (a - c) GO-term enrichment analysis of (a) 24 hours PL MpSYP12A interactome, (b) 24 hours PL MpSYP13B interactome, and (c) measured whole proteome. The top 20 overrepresented GO-terms are shown. (d) M. polymorpha homologs of AtSYP1-interacting proteins are highlighted in black or grey on volcano plots of 24 hours PL. Venn diagram shows numbers of potential interactors that are homologous to the AtSYP1interacors and their overlaps. (e) Potential interactors that are predicted to 
plots of 24 hours PL. Venn diagram shows numbers of the predicted plasma membrane-localized proteins and their overlaps.

Fig. 4 Potential interactors that preferentially interact with MpSYP12A or MpSYP13B. (a) Proteins that preferentially interacted with MpSYP12A or MpSYP13B are highlighted in turquoise and magenta, respectively. (b) Relative protein abundances based on $\mathrm{BAQQ}$ values are indicated by sizes of circles. Protein abundances in the samples of MpSYP12A, MpSYP13B, and wildtype Tak-1 are shown in turquoise, magenta, and dark grey, respectively. Proteins that were exclusively identified from the samples of MpSYP12A or MpSYP13B but not from the wildtype Tak-1 sample are annotated except for MpSYP12A and MpSYP13B.

Table 1 Potential interactors that preferentially interact with MpSYP12A or MpSYP13B. Proteins that are highlighted in turquoise or magenta in Fig. 4 are listed.

Fig. S1 Nucleotide and amino acid sequence of the miniTurbo biotin ligase used in this study. The nucleotide sequence of the original miniTurbo (Branon et al., 2018) was modified based on preferential codonusage of M. polymorpha. A linker sequence of 15 amino acids length was added to the C-terminus of miniTurbo, to minimize potential sterical impairments of the enzymatic function. The linker sequence was fused to a single Myc-tag peptide of 10 amino acids length, to enable immunoblot detection of miniTurbo-fusion proteins and affinity purification using MycTrap.

Fig. S2 Plasmid maps of the binary vectors used in this study. (a) Vector map of pMKMM23 for the expression of miniTurbo-Myc-MpSYP12A. (b) Vector map of pMKMM22 for the expression of miniTurbo-MycMpSYP13B.

Fig. S3 Evaluation of the fusion protein expression, biotin depletion methods, and Myc-Trap Co-IP by immunoblotting. (a) Selection of transgenic lines expressing miniTurbo-Myc-MpSYP12A and miniTurboMyc-MpSYP13B fusion-proteins. Immunoblot (IB) of cell extracts from 10day old thalli. MiniTurbo-Myc fusion proteins were detected by using an anti-Myc antibody. Cell extract of wildtype Tak-1 (WT) was used as a control. (b and c) Comparison of biotin depletion methods. Ten-day old thalli of a transgenic line expressing miniTurbo-Myc-MpSYP13B and 
wildtype Tak-1 (WT) were treated with $700 \mu \mathrm{M}$ biotin solution for 24 hours. Total protein was extracted (input) and free biotin was removed from the

1230

1231

1232

1233

1234

1235

1236

1237

1238

1239

1240

1241

1242

1243

1244

1245

1246

1247

1248

1249

1250

1251

1252

1253

1254

1255

1256

1257

1258

1259

1260

1261

1262

1263 samples by methanol:choloform precipitation (IP_1), PD-10 column desalting (IP_2), or Zeba spin column desalting (IP_3) before pulldown of biotinylated proteins using streptavidin-agarose beads. Streptavidin (SA) Immunoblots (IB) of biotinylated proteins. (b) Biotinylated proteins in cell extracts (input) and IP-eluates (IP-1, IP_2, IP_3) for the different biotin depletion methods. (c) Biotinylated proteins in cell extract (input), the supernatant of the streptavidin-agarose beads after affinity pulldown (unbound_1, unbound_2, unbound_3), and IP-eluates (IP_1, IP_2, IP_3) for the different biotin depletion methods. All depletion methods were tested in duplicates. (d) Evaluation of Myc-Trap Co-IP by immunoblotting. Immunoblot (IB) of cell extracts from 10-day old thalli. MiniTurbo-Myc fusion proteins were detected by using an anti-Myc antibody in cell extracts (input) and IP-eluates (IP) after affinity-purification using Myc-Trap beads. Coomassie Brilliant Blue-stained (CBB) membranes are shown as loading controls.

Fig. S4 Biotin ligase activity in M. polymorpha. Streptavidin (SA) immunoblots (IB) of cell extracts from $M$. polymorpha transgenic lines expressing miniTurbo-Myc-MpSYP12A (upper panels) and miniTurboMyc-MpSYP13B (lower panels) that were (a) treated with 0-700 $\mu \mathrm{M}$ biotin solutions for 24 hours at RT or (b) treated with $700 \mu \mathrm{M}$ biotin solution for 0-6 hours at RT. Cell extracts of wildtype Tak-1 (WT) treated with $700 \mu \mathrm{M}$ biotin solution for (a) 24 hours or (b) 6 hours were used as controls. Arrows indicate the positions of the biotinylated miniTurbo-Myc-MpSYP12A and miniTurbo-Myc-MpSYP13B fusion-proteins. Coomassie Brilliant Bluestained $(\mathrm{CBB})$ membranes are shown as loading controls.

Fig. S5 GO-term enrichment analysis. GO-term enrichment analysis of (a) 4 hours PL MpSYP12A interactome, (b) 4 hours PL MpSYP13B interactome, and (c) measured whole proteome. The top 20 overrepresented GO-terms are shown.

Fig. S6 Uncropped images of immunoblots used in figures. 


\section{Figure 1}

(a)

\begin{tabular}{|l|l|l|l|l|}
\hline PMPSYP12A (3.5 kb genomic) & 5'UTR & miniTurbo-Myc & MPSYP12A & 3'UTR \\
\hline pMPSYP13B (3.5 kb genomic) & 5'UTR $^{\prime}$ miniTurbo-Myc & MPSYP13B & 3'UTR \\
\hline
\end{tabular}

(b)

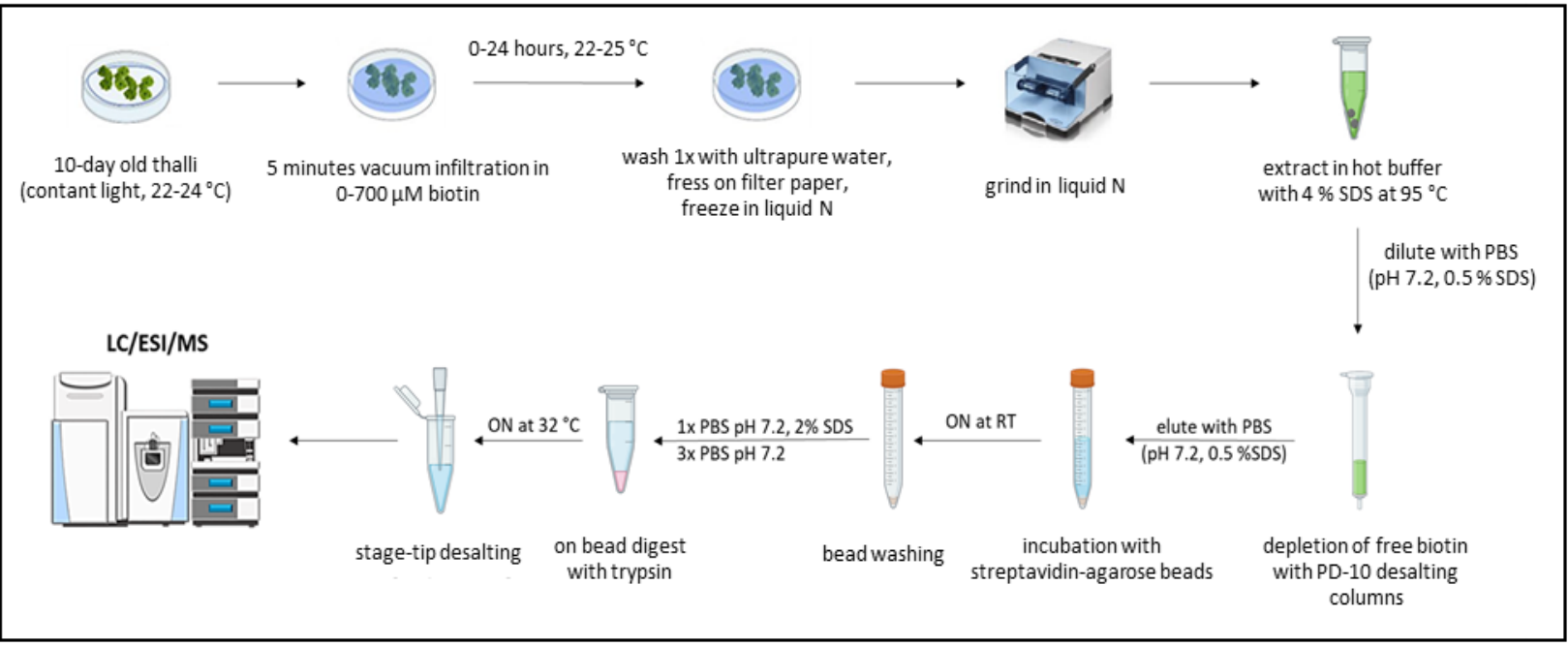

(c)

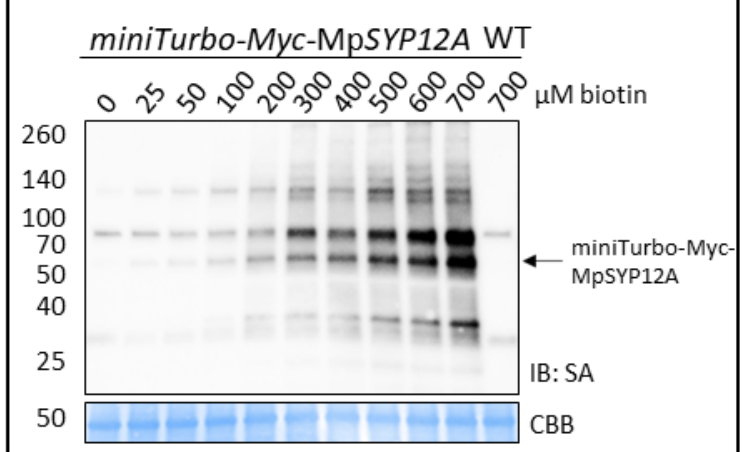

miniTurbo-Myc-MpSYP13B WT

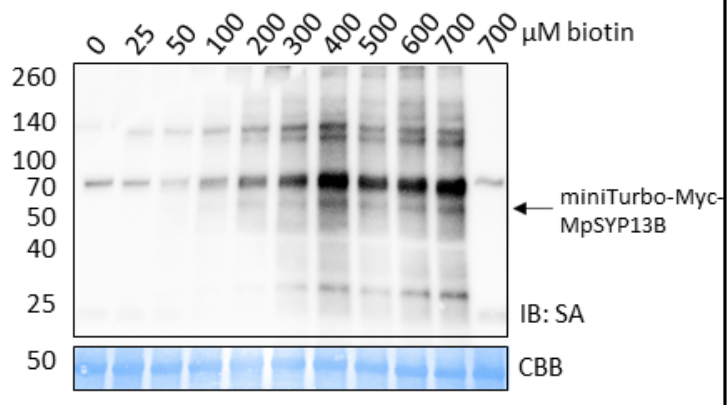

(d)

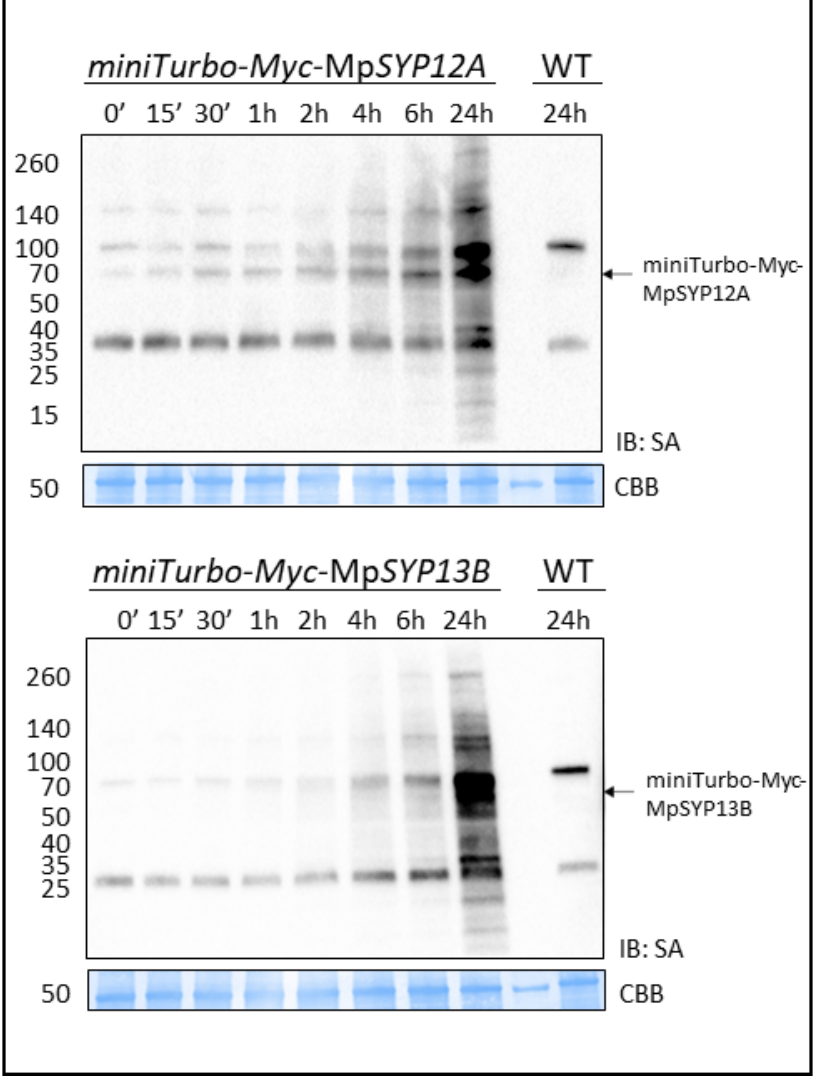




\section{Figure 2}

(a)

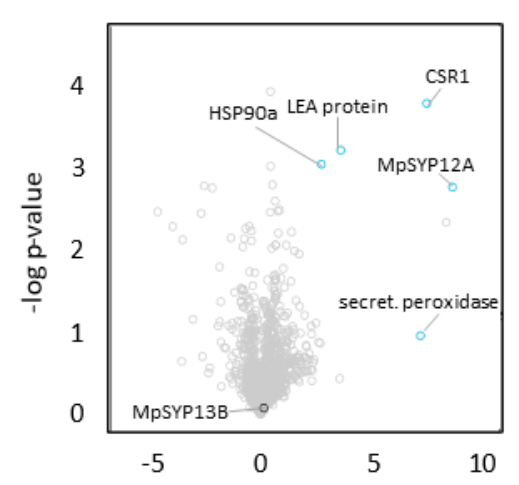

log2 fold-change [SYP12A/Tak-1]

(b)

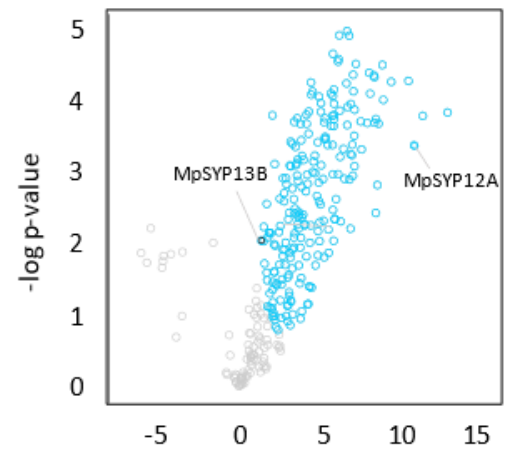

log2 fold-change [SYP12A/Tak-1]

(c)

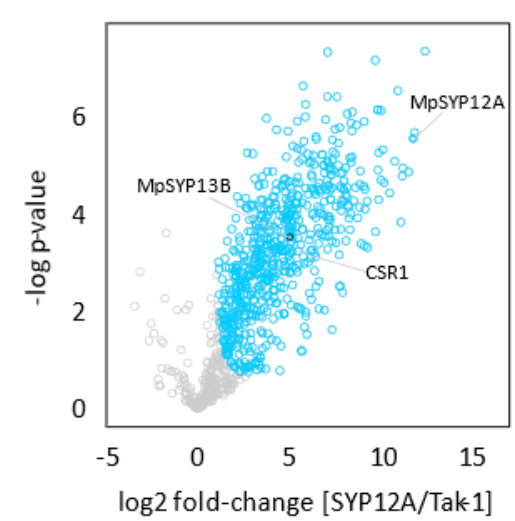

(d)

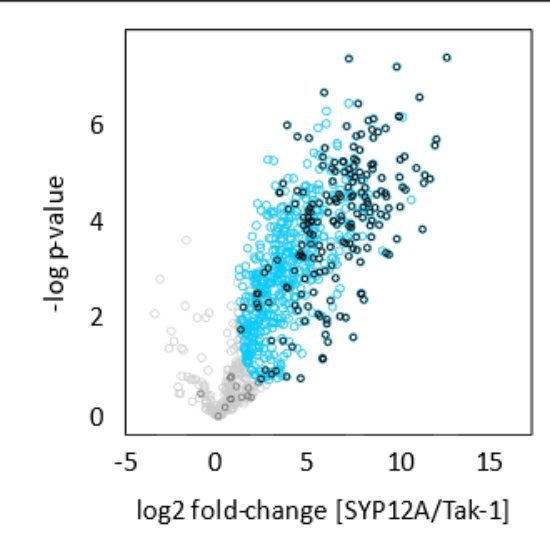

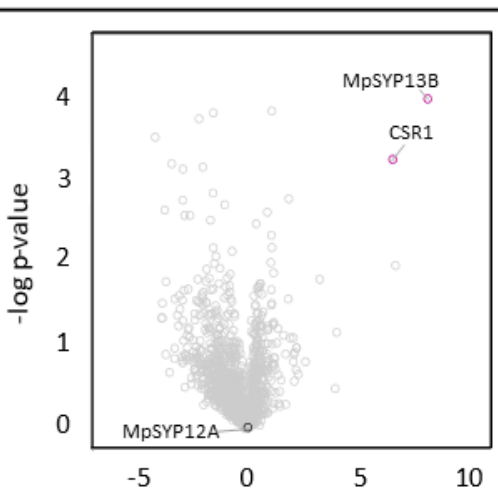

not significant

- sign. SYP12A

- sign. SYP13B

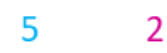

$\begin{array}{lll}4 & 1 & 1\end{array}$

$\log 2$ fold-change [SYP13B/Tak-1]

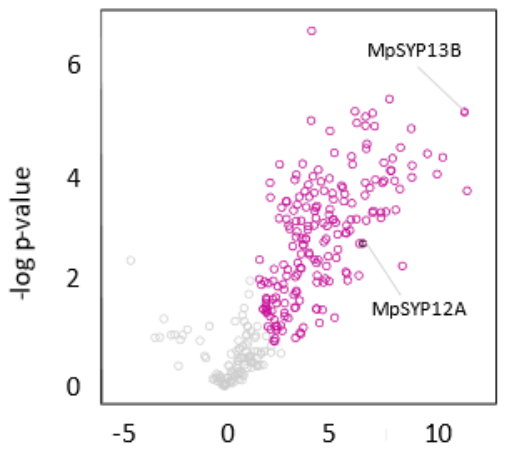

not significant

sign. SYP12A

- sign. SYP13B

$215 \quad 190$

$\begin{array}{lll}32 & 183 & 7\end{array}$

$\log 2$ fold-change [SYP13B/Tak-1]

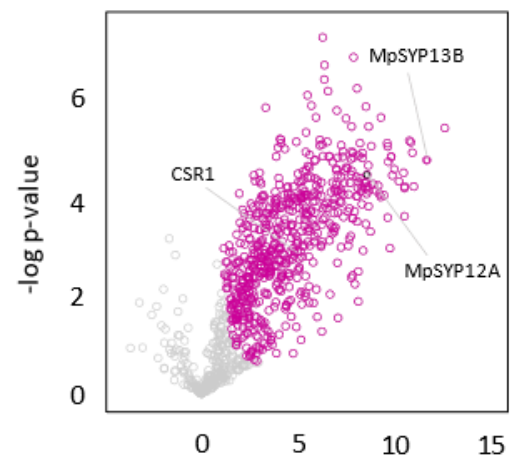

not significant

sign. SYP12A

- sign. SYP13B

637

602

7056735

log2 fold-change [SYP13B/Tak-1]

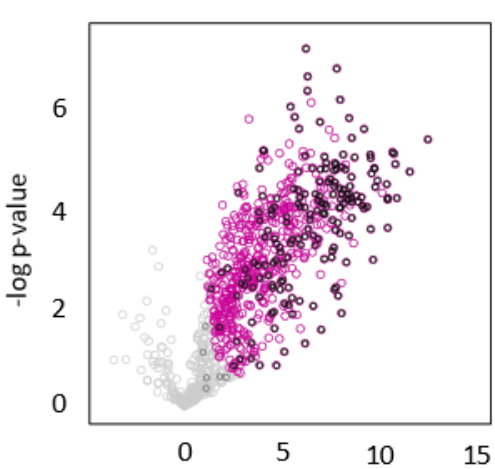

log2 fold-change [SYP13B/Tak-1] not significant

sign. SYP12A

- sign. SYP13B

overlaps

- sign. overlaps

$24 \mathrm{~h}$

$637 \quad 602$

\begin{tabular}{c|c}
\hline 446 & 430 \\
191 & 172 \\
\hline 24 & 18 \\
\hline 215 & 190 \\
\hline
\end{tabular}

$4 \mathrm{~h}$ 
(a)

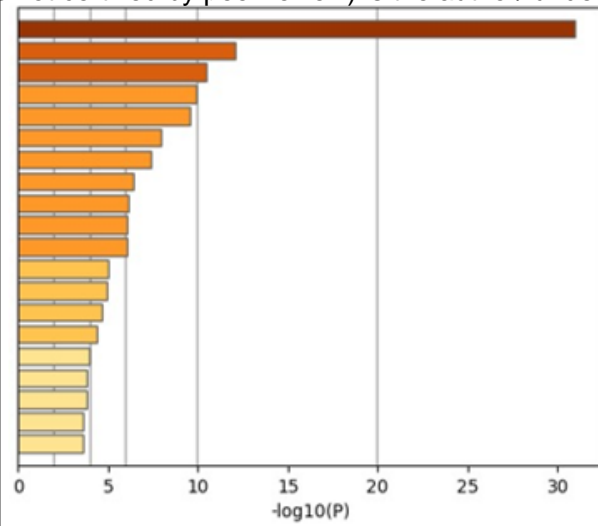

O-0016192: vesicle-mediated transport ath04144: Endocytosis

897: endocytosis

GO:0061024: membrane organization

GO:0010256: endomembrane system organization GO:0051640: organelle localization

GO:0097435: supramolecular fiber organization

GO:0000904: cell morphogenesis involved in differentiation GO:0048193: Golgi vesicle transport

GO:0006887: exocytosis

GO:0051130: positive regulation of cellular component organization

GO:0090626: plant epidermis morphogenesis

GO:0010118: stomatal movement

GO:0051668: localization within membrane

GO:0046777: protein autophosphorylation

GO:0071219: cellular response to molecule of bacterial origin

GO:0007009: plasma membrane organization

GO:0060321: acceptance of pollen

GO:0000911: cytokinesis by cell plate formation

GO:0009617: response to bacterium (b)

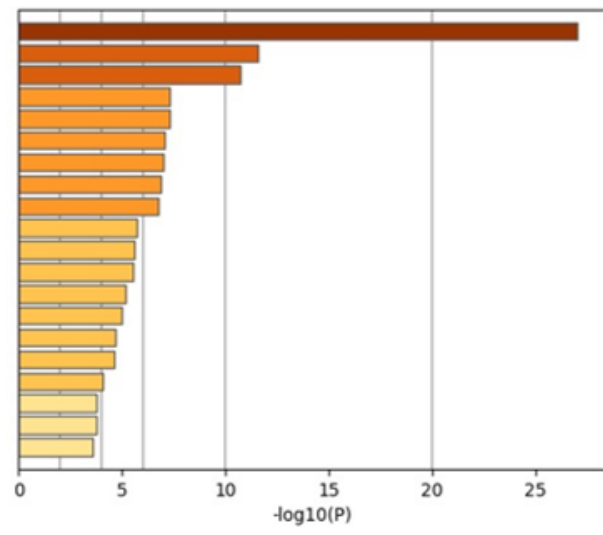

GO:0016192: vesicle-mediated transport ath04144: Endocytosis

GO:0006897: endocytosis

GO:0016197: endosomal transport

GO:0006887: exocytosis

GO:0016050: vesicle organization

GO:0007010: cytoskeleton organization

GO:0098876: vesicle-mediated transport to the plasma membrane

GO:0051640: organelle localization

GO:0000902: cell morphogenesis

GO:0010256: endomembrane system organization

GO:0051668: localization within membrane

GO:0051668: localization within mem

G.0010118: stomatal movement

cellar component organization

G0 0051130: positive regulation of cellular component organization GO:0046777: protein autophosphorylation

GO:0071219: cellular response to molecule of bacterial origin

GO:0000911: cytokinesis by cell plate formation GO:0090626: plant epidermis morphogenesis GO:0009606: tropism (c)

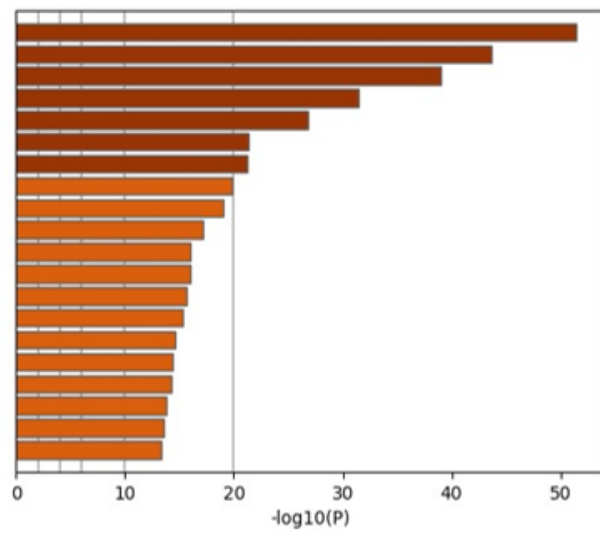

GO:0006520: cellular amino acid metabolic process ath01200: Carbon metabolism

GO:0006091: generation of precursor metabolites and energy GO:0046907: intracellular transport ath03010: Ribosome

GO:0009259: ribonucleotide metabolic process GO:0009259: ribonucleotide metabolic GO:0043038: amino acid activation

GO:0009657: plastid organization GO:0032787: monocarboxylic acid metabolic proces GO:0044282: small molecule catabolic process GO:0065003: protein-containing complex assembly GO:0043648: dicarboxylic acid metabolic

GO:0009793: embryo development ending in seed dormancy ath00710: Carbon fixation in photosynthetic organisms ath00010: Glycolysis / Gluconeogenesis ath00630: Glyoxylate and dicarboxylate metabolism GO:0009064: glutamine family amino acid metabolic process th03050: Proteasome

GO:0061024: membrane organization

(d)

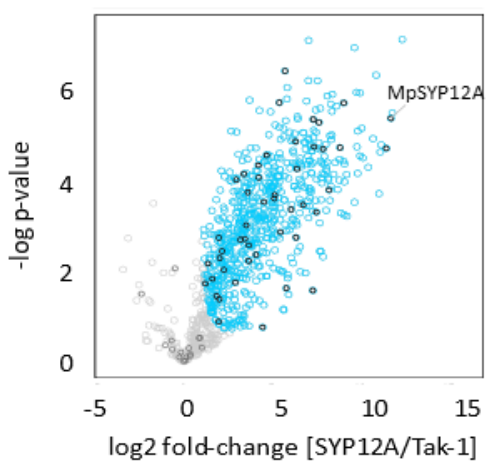

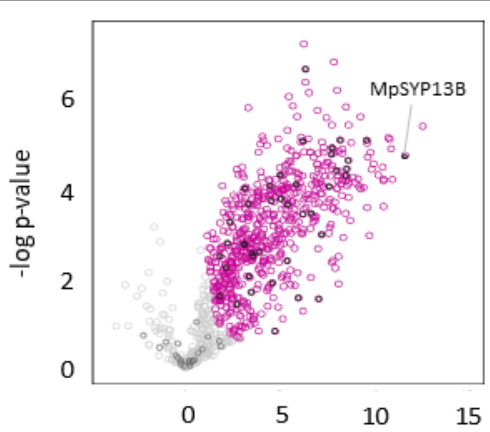

log2 fold-change [SYP13B/Tak-1] not significant

sign. SYP12A

sign. SYP13B

overlaps

- sign. overlaps

$47 / 637$

$\begin{array}{lll}8 & 39 & 1\end{array}$

$40 / 602$ (e)

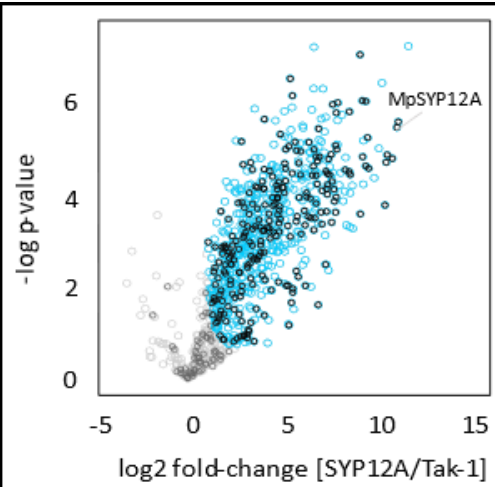

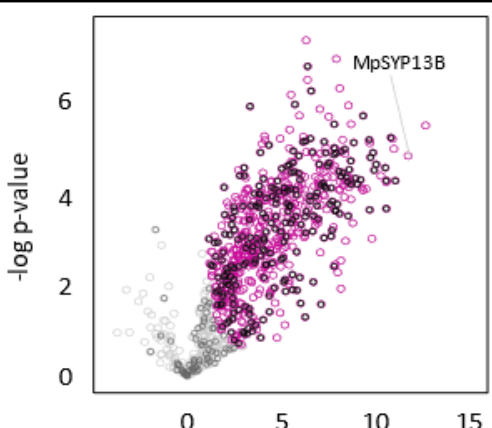

not significant sign. SYP12A - sign. SYP13B

overlaps

sign. overlaps

$242 / 602$

172256 $231 / 602$

$\log 2$ fold-change [SYP13B/Tak-1] 


\section{Figure 4}

(a)

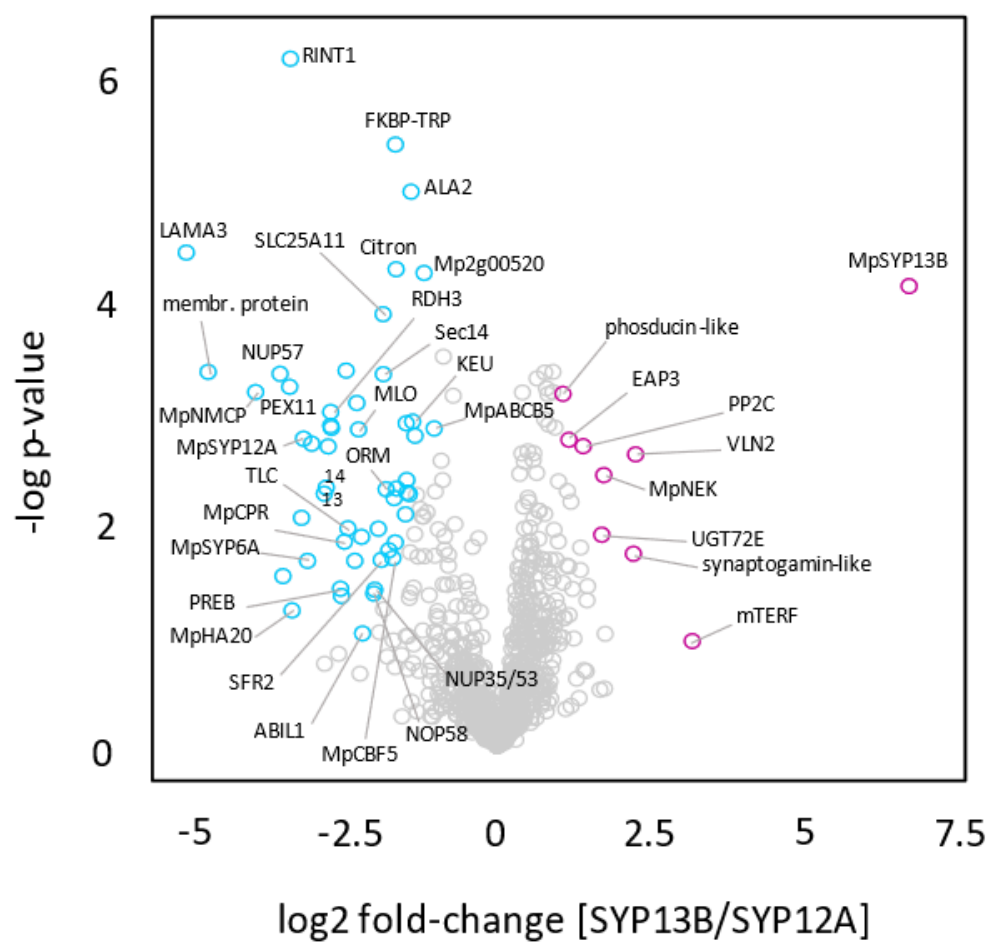

not significant

sign. SYP12A

- sign. SYP13B

- Tak-1 (WT)

(b)

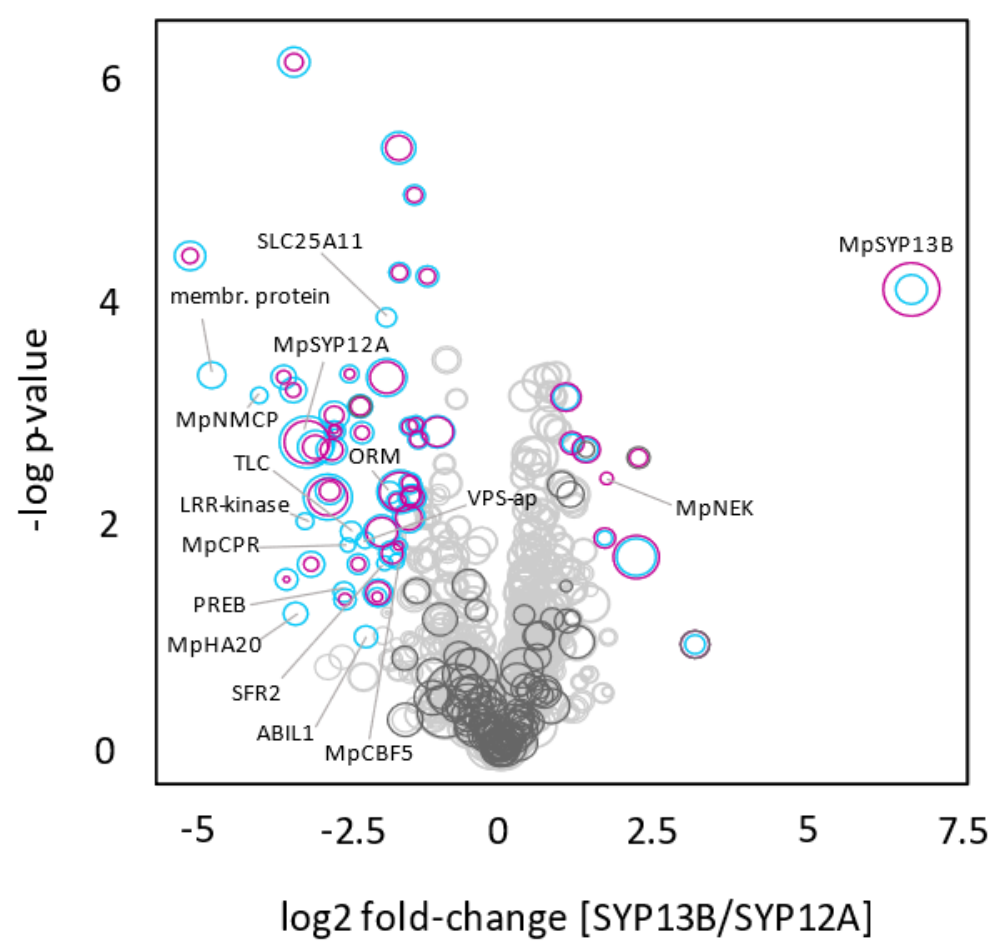

relative $\mathrm{iBAQ}$

- 2

○ 5

O 8.5

○ 10

not significant

sign. SYP12A

- sign. SYP13B

- Tak-1 (WT)

log2 fold-change [SYP13B/SYP12A] 
(which was not certified by peer review) is the author/funder. All rights reserved. No reuse allowed without permission.

Table 1

\begin{tabular}{|c|c|c|c|c|c|c|}
\hline No. & MpGene ID & $\begin{array}{l}\text { A. thaliana } \\
\text { homolog } \\
\text { (AGI code) }\end{array}$ & Annotation & $\begin{array}{l}\text { Log2 fold-change } \\
\text { SYP13B / SYP12A }\end{array}$ & $\begin{array}{c}-\log \\
\text { p-value }\end{array}$ & Exclusive \\
\hline 1 & Mp1g19980 & - & LAMA3 & $-5,05$ & 4,41 & no \\
\hline 2 & Mp2g10520 & AT4G31080 & membr. Protein & $-4,69$ & 3,34 & MpSYP12A \\
\hline 3 & Mp3g21160 & AT1G67230 & MpNMCP & $-3,92$ & 3,16 & MpSYP12A \\
\hline 4 & Mp3g05990 & AT3G10650 & NUP57 & $-3,52$ & 3,32 & no \\
\hline 5 & Mp1g10960 & - & Mp1g10960 & $-3,48$ & 1,52 & no \\
\hline 6 & Mp1g28560 & AT1G47750 & PEX11 & $-3,37$ & 3,21 & no \\
\hline 7 & $\mathrm{Mp} 6 \mathrm{~g} 02820$ & AT3G47700 & RINT1 & $-3,36$ & 6,14 & no \\
\hline 8 & Mp3g12450 & AT4G30190 & MpHA20 & $-3,33$ & 1,21 & MpSYP12A \\
\hline 9 & Mp2g17240 & AT5G01950 & LRR-kinase & $-3,18$ & 2,04 & MpSYP12A \\
\hline 10 & Mp6g00050 & AT1G61290 & MpSYP12A & $-3,14$ & 2,74 & no \\
\hline 11 & Mp3g18380 & AT1G28490 & MpSYP6A & $-3,08$ & 1,65 & no \\
\hline 12 & Mp1g08670 & AT1G73200 & TEX2 & $-3,01$ & 2,70 & no \\
\hline 13 & Mp6g00330 & AT4G04910 & NSF & $-2,81$ & 2,25 & no \\
\hline 14 & Mp2g14450 & AT5G45420 & MpMYB3 & $-2,77$ & 2,31 & no \\
\hline 15 & $\mathrm{Mp} 7 \mathrm{~g} 04560$ & AT3G55420 & AT3G55420-like & $-2,74$ & 2,68 & no \\
\hline 16 & Mp2g20600 & AT5G43360 & PHO84 & $-2,70$ & 2,86 & no \\
\hline 17 & Mp1g14480 & AT5G45160 & RDH3/SEY1 & $-2,70$ & 2,98 & no \\
\hline 18 & Mp3g07700 & AT5G62670 & MpHA11 & $-2,69$ & 2,84 & no \\
\hline 19 & Mp7g14000 & AT3G52190 & PREB & $-2,54$ & 1,41 & MpSYP12A \\
\hline 20 & Mp7g10500 & AT1G20970 & AT1G20970-like & $-2,53$ & 1,34 & no \\
\hline 21 & Mp1g28850 & AT5G64930 & MpCPR & $-2,48$ & 1,82 & MpSYP12A \\
\hline 22 & $\mathrm{Mp} 7 \mathrm{~g} 07570$ & AT1G48090 & VPS-associated & $-2,45$ & 3,35 & no \\
\hline 23 & Mp2g19670 & AT1G21790 & TLC lipid-sens. & $-2,42$ & 1,94 & no \\
\hline 24 & Mp3g14570 & AT1G73020 & ANO10 & $-2,31$ & 1,65 & no \\
\hline 25 & Mp1g21390 & AT3G18610 & NSR1 & $-2,28$ & 3,06 & no \\
\hline 26 & Mp5g01540 & AT5G53760 & MLO & $-2,25$ & 2,83 & no \\
\hline 27 & Mp3g11440 & AT1G48090 & VPS-associated & $-2,20$ & 1,87 & MpSYP12A \\
\hline 28 & $\mathrm{Mp} 3 \mathrm{~g} 23010$ & AT2G46225 & ABIL1 & $-2,19$ & 1,01 & MpSYP12A \\
\hline 29 & $\mathrm{Mp} 2 \mathrm{~g} 00370$ & AT3G05060 & NOP58 & $-2,00$ & 1,36 & MpSYP12A \\
\hline 30 & Mp1g11950 & AT3G16310 & NUP35/53 & $-1,99$ & 1,40 & no \\
\hline 31 & Mp7g06360 & AT3G11950 & TRAF zinc finger & $-1,93$ & 1,94 & no \\
\hline 32 & Mp2g17840 & AT3G06510 & SFR2 & $-1,88$ & 1,66 & MpSYP12A \\
\hline 33 & Mp3g16790 & AT5G19760 & SLC25A11 & $-1,85$ & 3,86 & MpSYP12A \\
\hline 34 & Mp4g16700 & AT1G30690 & SEC14-related & $-1,85$ & 3,32 & no \\
\hline 35 & $\mathrm{Mp} 2 \mathrm{~g} 02640$ & AT1G01230 & ORM & $-1,80$ & 2,29 & MpSYP12A \\
\hline 36 & Mp2g10340 & AT3G12490 & CYSB-like & $-1,76$ & 1,75 & no \\
\hline 37 & Mp4g12650 & AT3G57150 & MpCBF5 & $-1,69$ & 1,68 & MpSYP12A \\
\hline 38 & Mp6g17400 & AT3G20500 & APC7 & $-1,67$ & 2,21 & no \\
\hline 39 & Mp6g16270 & AT2G45540 & BEACH & $-1,65$ & 1,82 & no \\
\hline 40 & $\mathrm{Mp} 8 \mathrm{~g} 00690$ & AT5G21990 & FKBP-TRP & $-1,65$ & 5,37 & no \\
\hline 41 & Mp3g00870 & AT5G13560 & Citron & $-1,64$ & 4,26 & no \\
\hline 42 & Mp4g16720 & AT1G30690 & SEC14-related & $-1,63$ & 2,30 & no \\
\hline 43 & Mp5g13890 & AT2G39280 & Ypt-activating & $-1,49$ & 2,07 & no \\
\hline 44 & $\mathrm{Mp} 8 \mathrm{~g} 06300$ & AT5G01180 & PTR5 & $-1,48$ & 2,88 & no \\
\hline 45 & Mp8g18220 & AT3G60860 & BIG & $-1,47$ & 2,38 & no \\
\hline 46 & $\mathrm{Mp} 4 \mathrm{~g} 23500$ & AT1G59820 & DRS2 & $-1,46$ & 2,26 & no \\
\hline 47 & Mp3g01730 & AT1G28250 & AT1G28250-like & $-1,43$ & 2,25 & no \\
\hline 48 & Mp1g04630 & AT5G44240 & ALA2 & $-1,40$ & 4,95 & no \\
\hline 49 & Mp6g07760 & AT1G12360 & $\mathrm{KEU}$ & $-1,37$ & 2,90 & no \\
\hline 50 & Mp5g23560 & AT3G62860 & Lysophospholipase & $-1,33$ & 2,77 & no \\
\hline 51 & $\mathrm{Mp} 2 \mathrm{~g} 00520$ & - & $\mathrm{Mp} 2 \mathrm{~g} 00520$ & $-1,19$ & 4,22 & no \\
\hline 52 & $\mathrm{Mp} 4 \mathrm{~g} 07450$ & AT3G28860 & MpABCB5 & $-1,02$ & 2,84 & no \\
\hline 53 & Mp6g13120 & AT5G14240 & phosducin-like & 1,07 & 3,14 & no \\
\hline 54 & Mp3g04380 & AT3G09030 & EAP3 & 1,17 & 2,74 & no \\
\hline 55 & Mp2g17470 & AT1G68410 & PP2C & 1,40 & 2,68 & no \\
\hline 56 & Mp2g15040 & AT4G01070 & UGT72E & 1,70 & 1,89 & no \\
\hline 57 & Mp5g04500 & AT3G04810 & MpNEK & 1,73 & 2,42 & MpSYP13B \\
\hline 58 & Mp6g00440 & AT1G22610 & Synaptogamin-like & 2,21 & 1,72 & no \\
\hline 59 & $\mathrm{Mp} 7 \mathrm{~g} 09750$ & AT2G41740 & VLN2 & 2,25 & 2,61 & no \\
\hline 60 & Mp3g14990 & AT4G14605 & mTERF & 3,17 & 0,94 & no \\
\hline 61 & Mp2g19600 & AT3G03800 & MpSYP13B & 6,69 & 4,11 & no \\
\hline
\end{tabular}




\section{Figure S1}

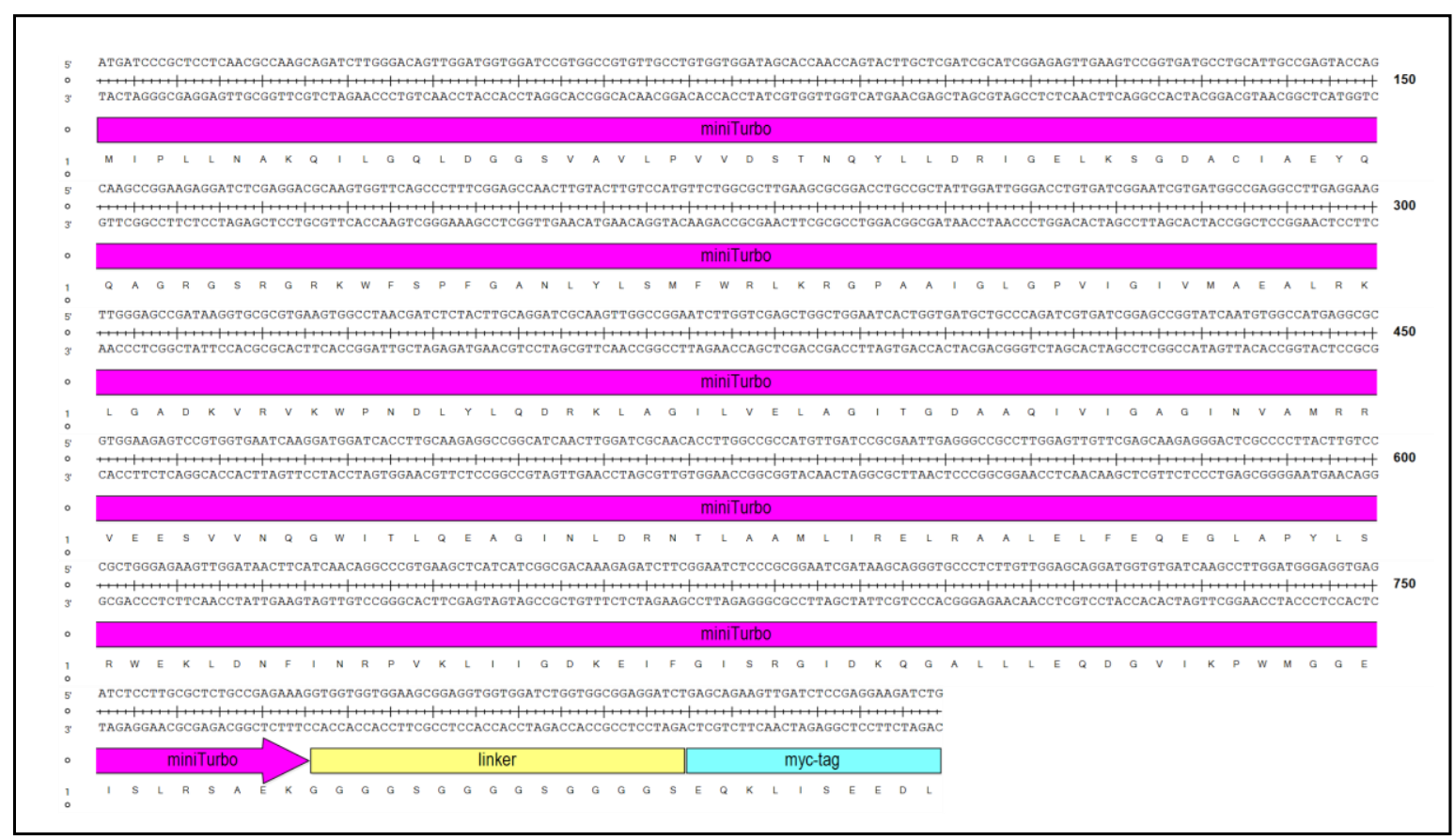




\section{Figure S2}

(a)

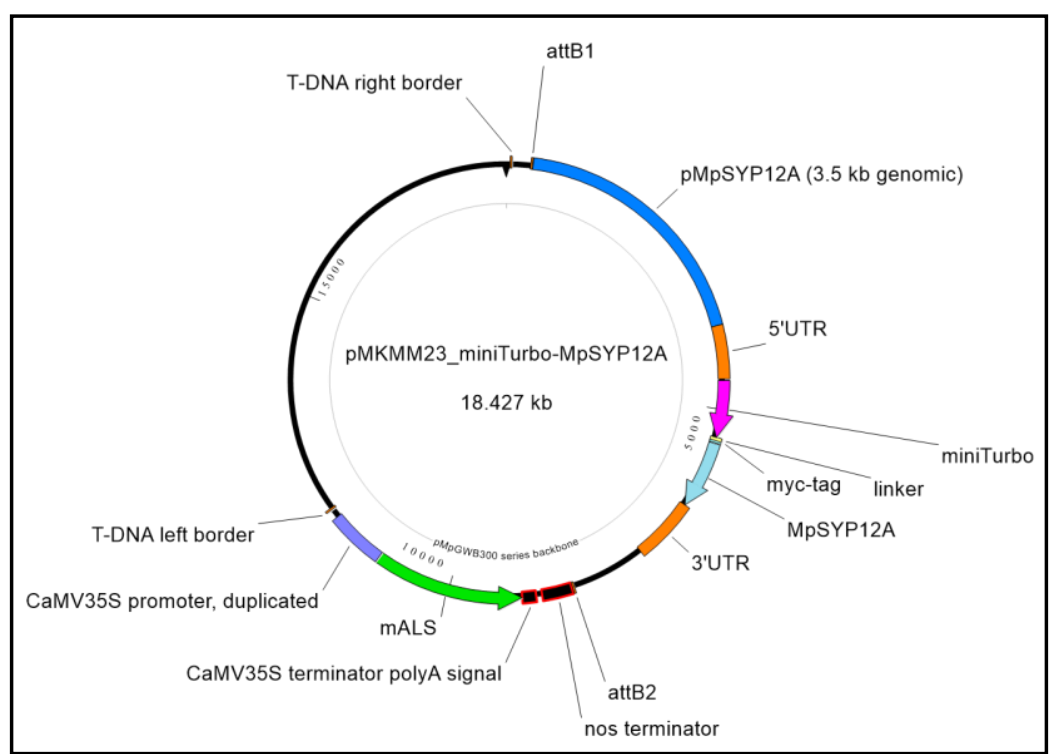

(b)

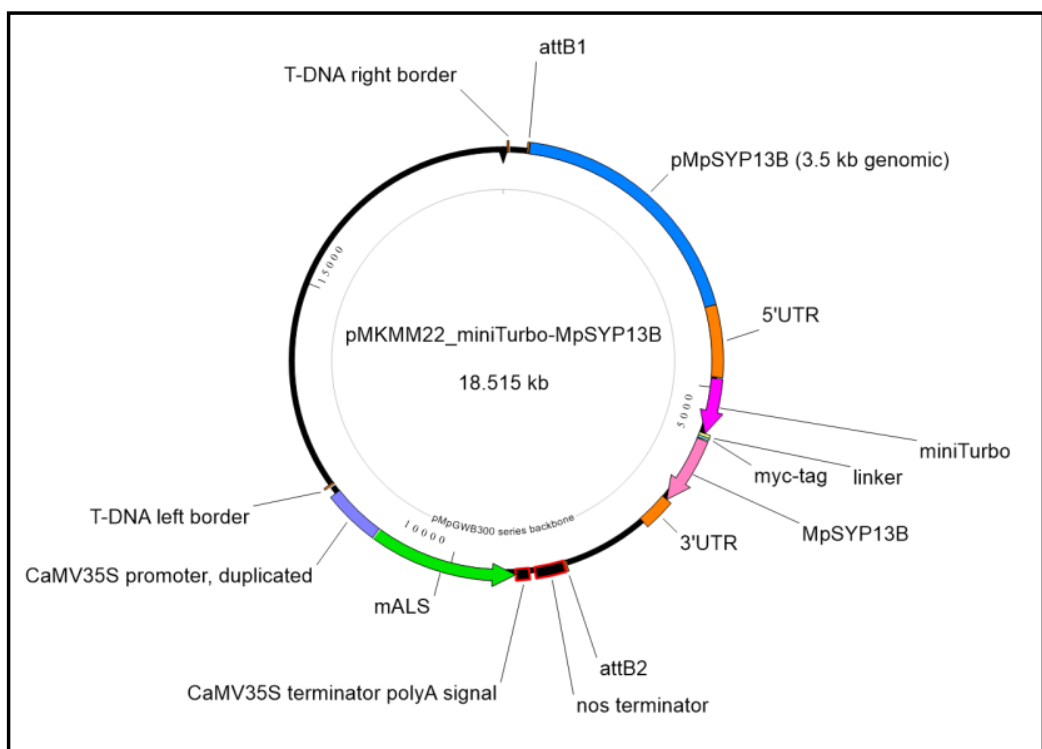




\section{Figure S3}

(a)

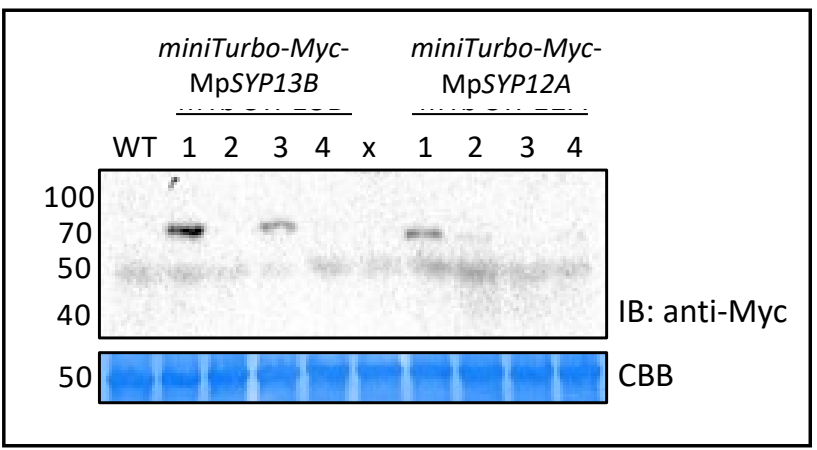

(b)

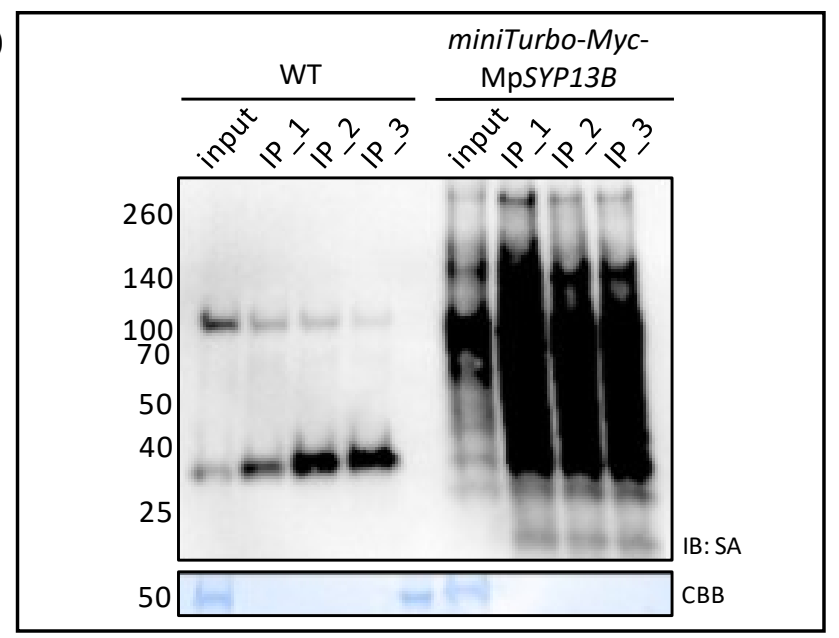

(c)

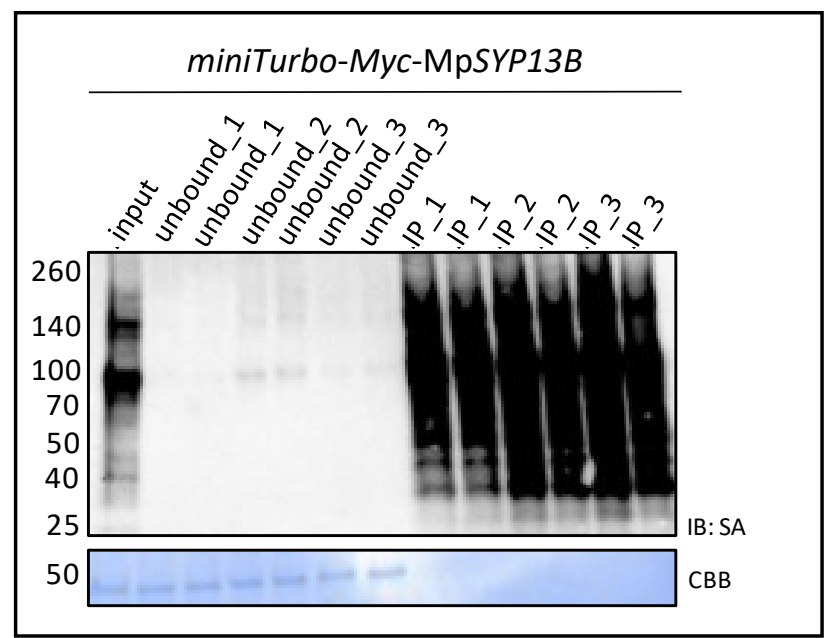

(d)

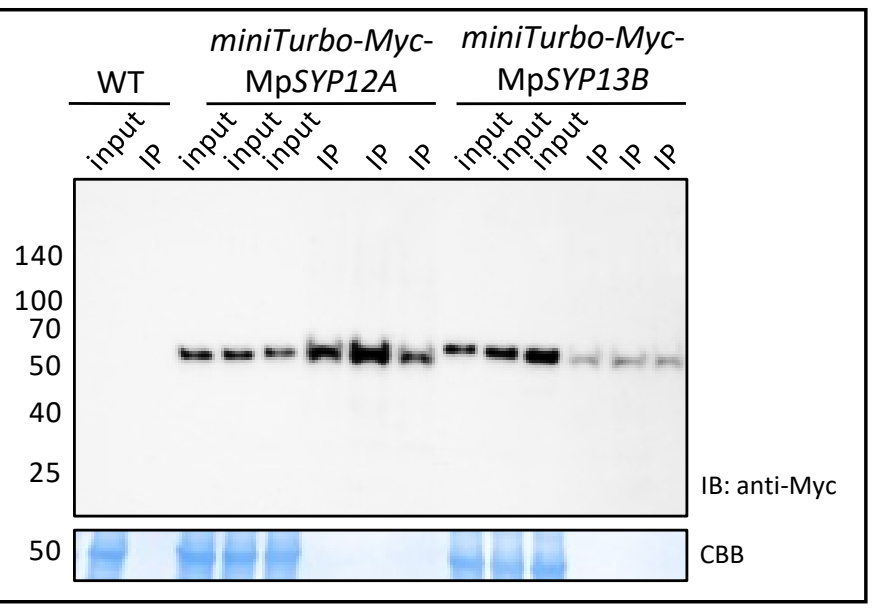




\section{Figure S4}

(a)
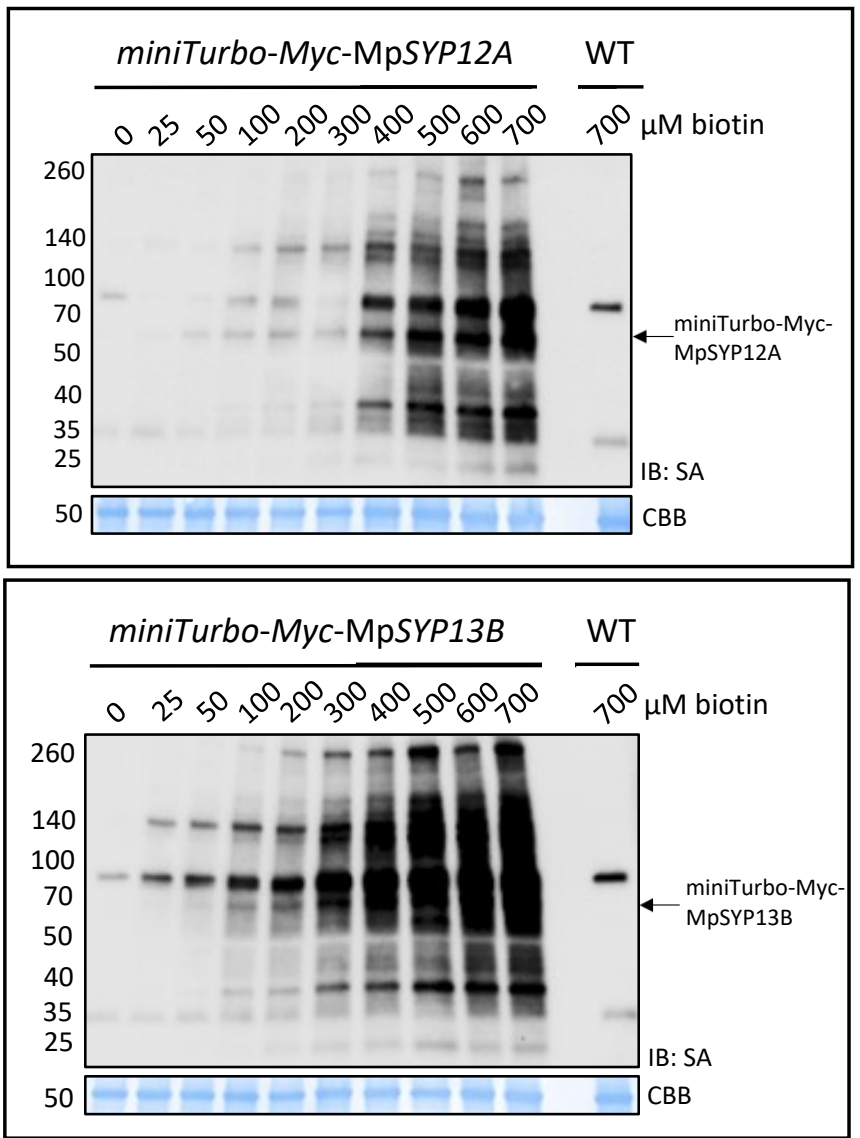

(b)
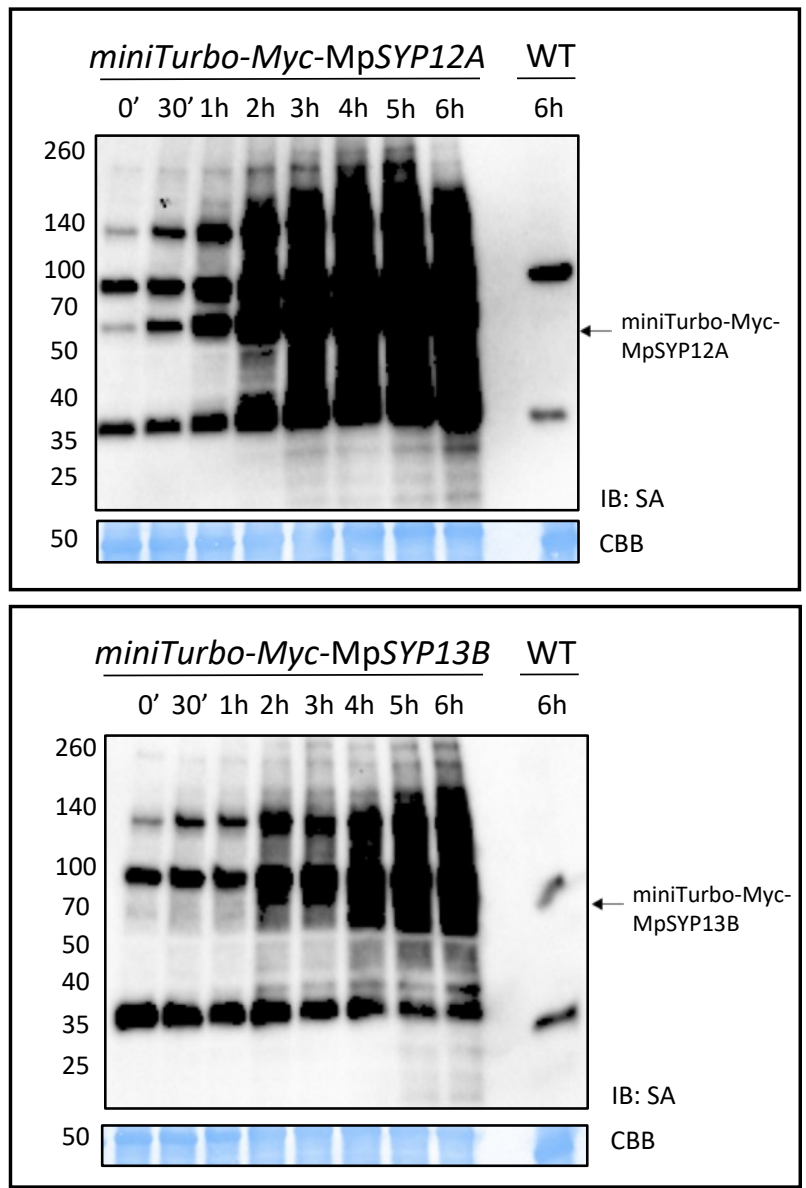


\section{Figure S5}

(a)

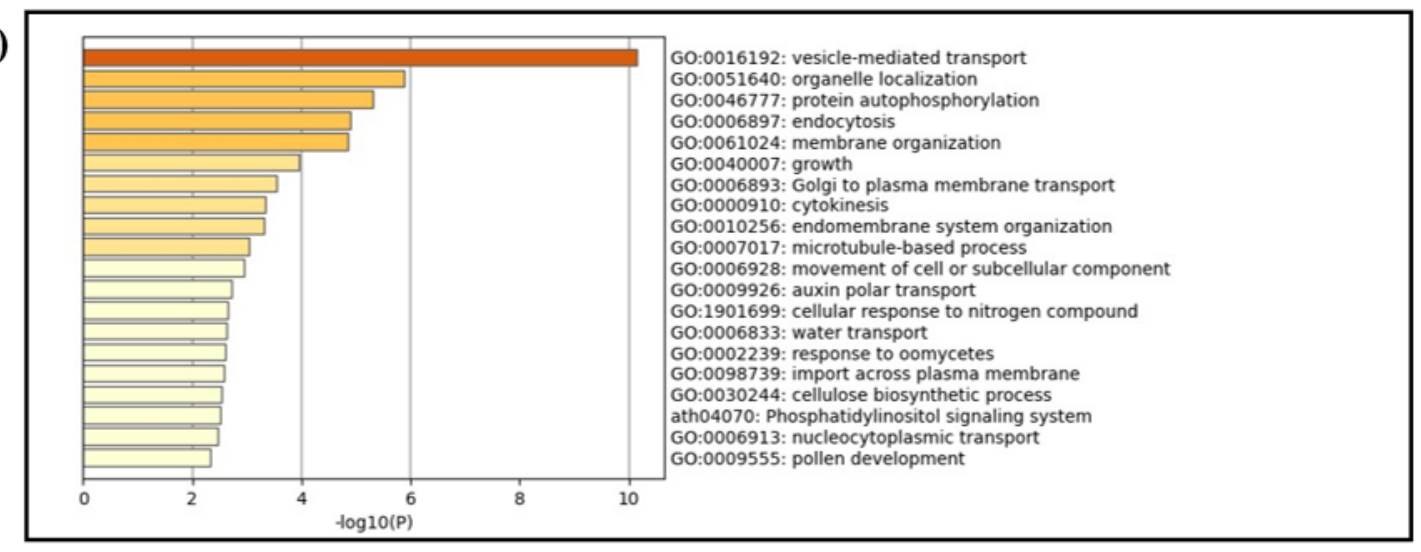

(b)

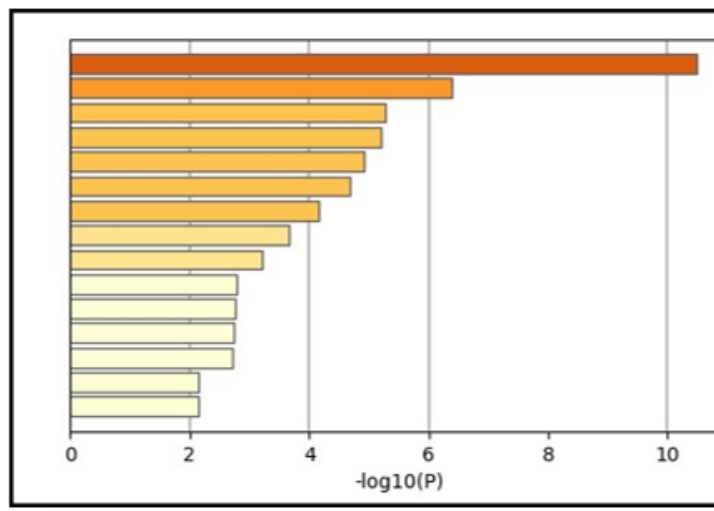

GO:0016192: vesicle-mediated transport GO:0051640: organelle localization

GO:0006897: endocytosis

GO:0048278: vesicle docking

GO:0046777: protein autophosphorylation

GO:0040007: growth

GO:0007017: microtubule-based process

GO:0000910: cytokinesis

GO:0006928: movement of cell or subcellular component GO:0006833: water transport

GO:0098739: import across plasma membrane

GO:0030244: cellulose biosynthetic process

ath04070: Phosphatidylinositol signaling system

GO:0009555: pollen development

GO:0072665: protein localization to vacuole (c)

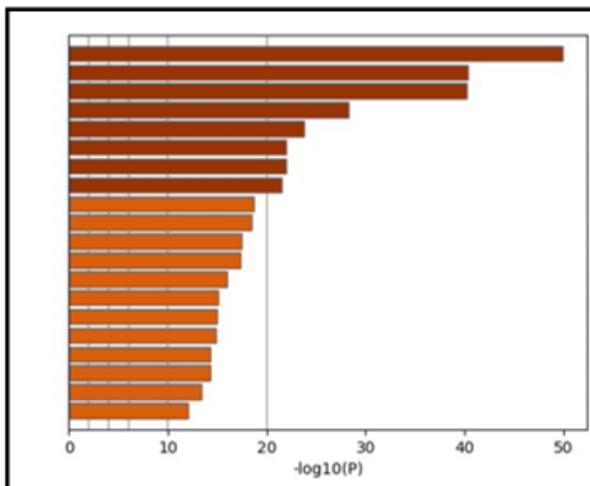

$60: 0006520$ : cellular amino acid metabolic process ath01200: Carbon metabolism

 co:0015031: protein tra

G0:0055086: nucleobase-containing small molecule metabolic process GO:0032787: monocarboxylic acid metabolic process

GO:0009657: plastid organization

GO:0044282: small molecule catabolic process

atho0190: Oxidative phosphorylation

60:0043648: dicarboxylic acid metabolic process

60.0043038: amino acid activation

位

ath00710: Carbon fixation in photosynthetic organisms

ath03050: Proteasome

ath00630: Glyoxylate and dicarboxylate metabolism

60.0046034 : ATP metabolic process

$60: 0006457$ : protein folding

60:0009266: glutamine family amino acid metabolic process

. 


\section{Figure S6}

\section{- uncropped immunoblots}

\section{Figure 1c}

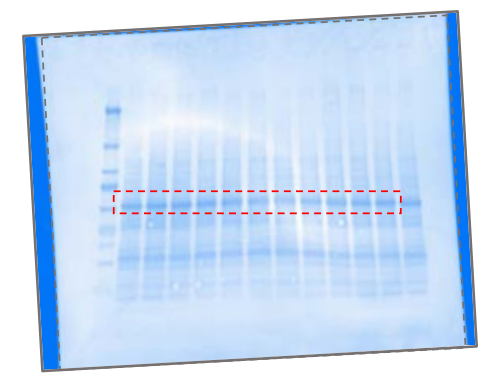

CBB stained membrane

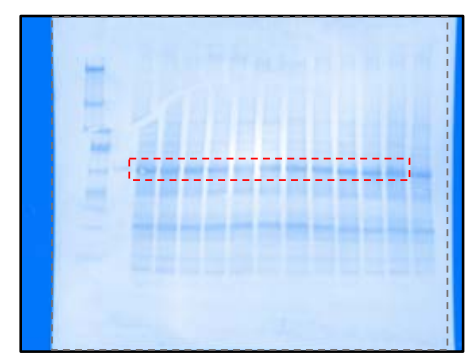

CBB stained membrane

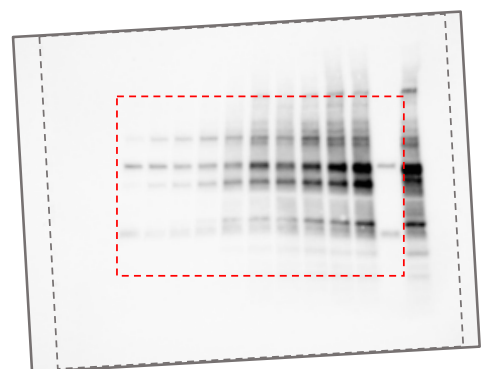

IB: streptavidin-HRP

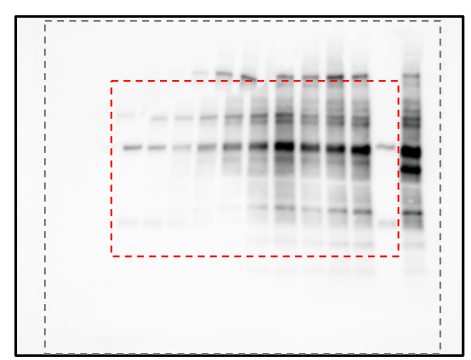

IB: streptavidin-HRP

\section{Figure 1d}

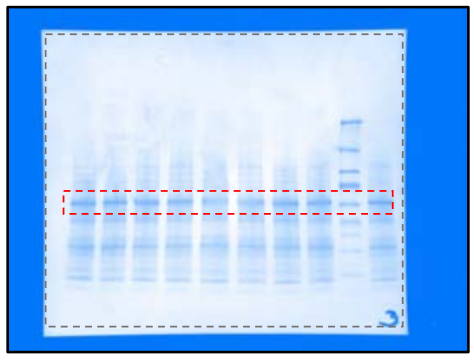

CBB stained membrane

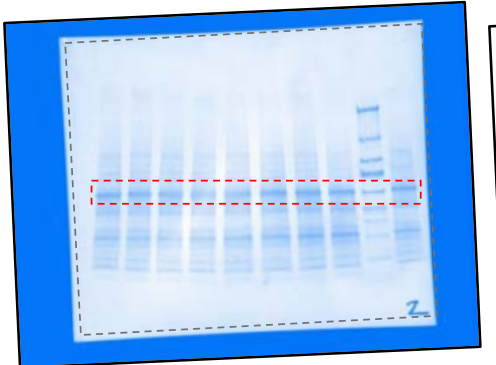

CBB stained membrane

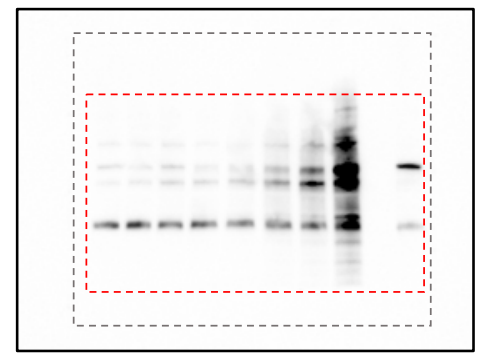

IB: streptavidin-HRP

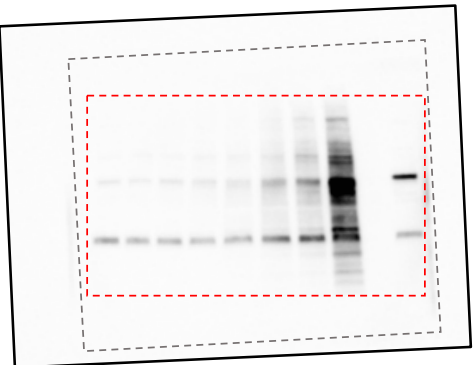

IB: streptavidin-HRP 


\section{Figure S3a}

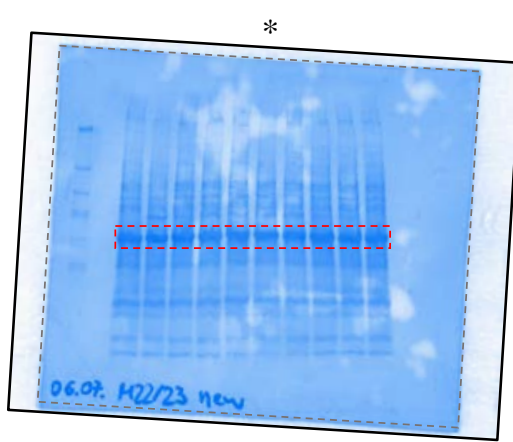

CBB stained membrane

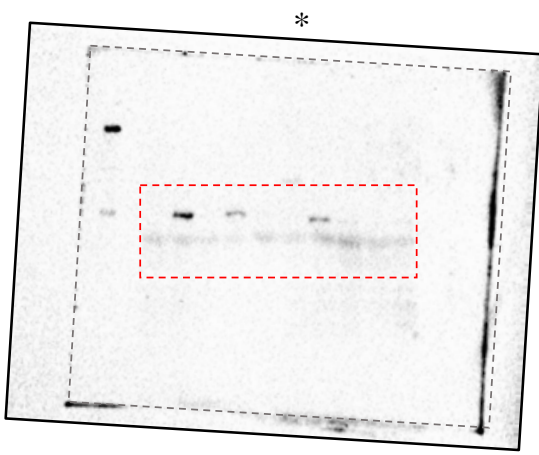

IB: anti-myc

* LYSM-mTb $=\mathrm{x}$

\section{Figure S3b}
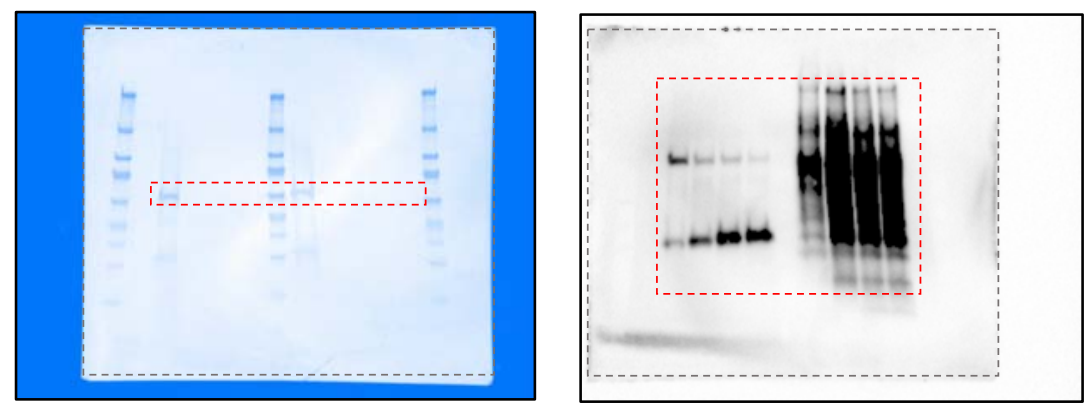

CBB stained membrane

IB: streptiavidin-HRP

\section{Figure S3c}

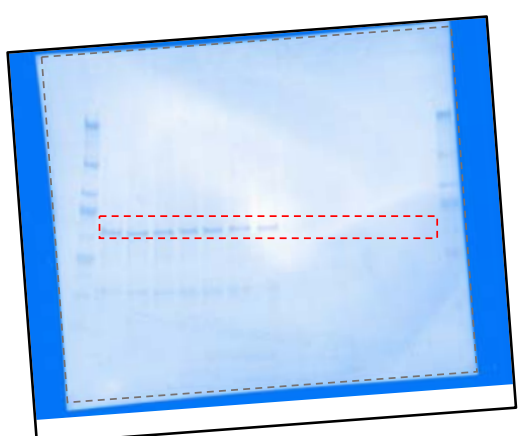

CBB stained membrane

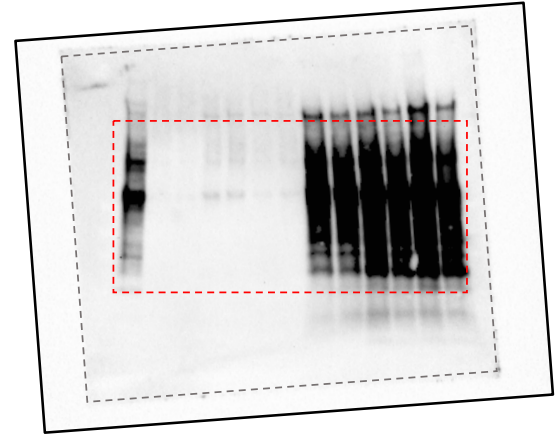

IB: streptiavidin-HRP

\section{Figure S3d}

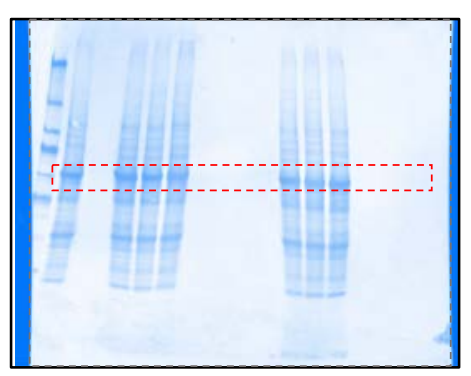

CBB stained membrane

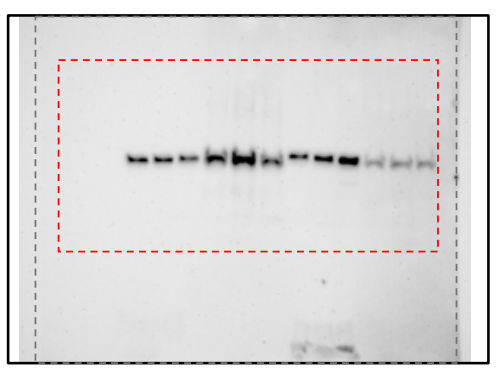

IB: anti-myc 


\section{Figure S4a}

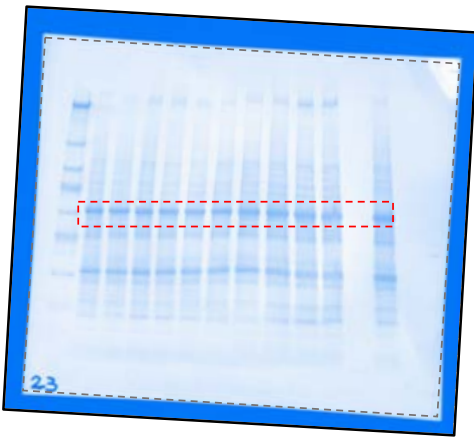

CBB stained membrane

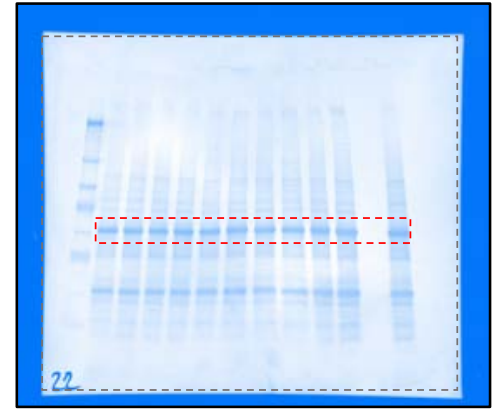

CBB stained membrane

\section{Figure S4b}

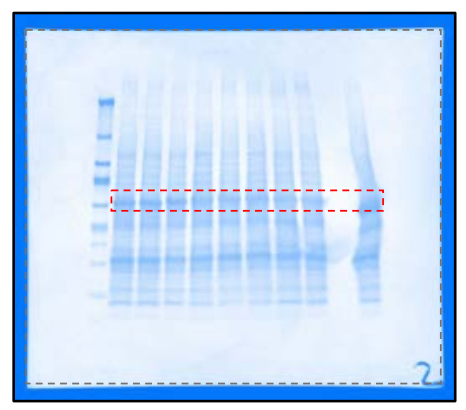

CBB stained membrane

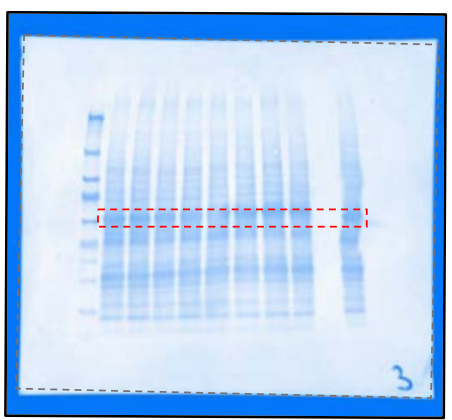

CBB stained membrane

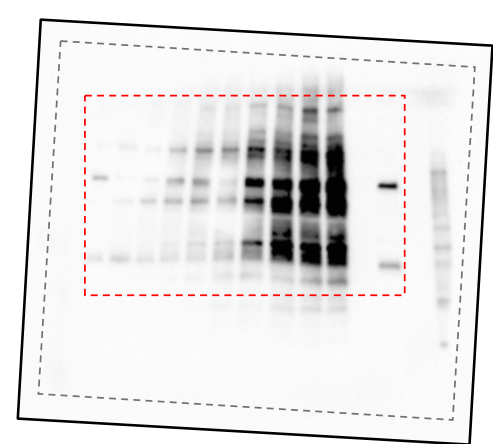

IB: streptavidin-HRP

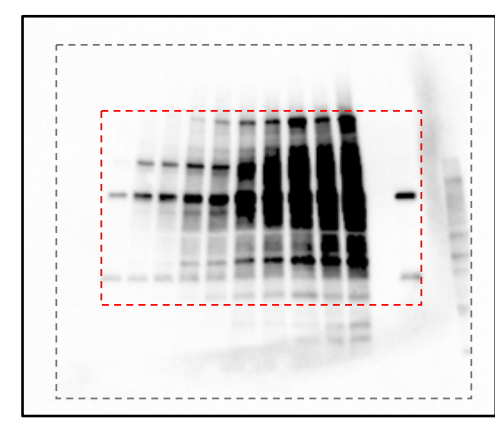

IB: streptavidin-HRP

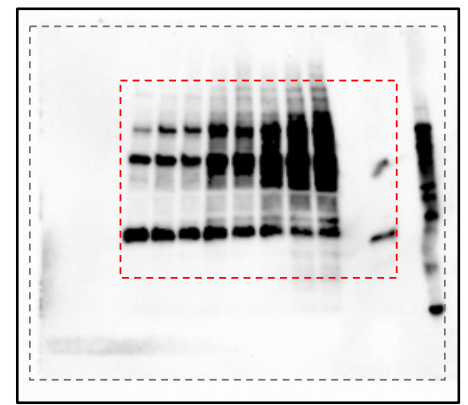

IB: streptavidin-HRP

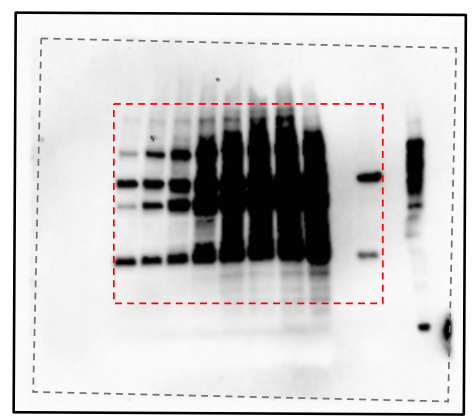

IB: streptavidin-HRP 